\title{
HOW TO GET A CONSERVATIVE WELL-POSED LINEAR SYSTEM OUT OF THIN AIR. PART II. CONTROLLABILITY AND STABILITY*
}

\author{
MARIUS TUCSNAK ${ }^{\dagger}$ AND GEORGE WEISS ${ }^{\ddagger}$
}

\begin{abstract}
Let $A_{0}$ be a possibly unbounded positive operator on the Hilbert space $H$, which is boundedly invertible. Let $C_{0}$ be a bounded operator from $\mathcal{D}\left(A_{0}^{1 / 2}\right)$ (with the norm $\|z\|_{1 / 2}^{2}=\left\langle A_{0} z, z\right\rangle$ ) to another Hilbert space $U$. In Part I of this work we have proved that the system of equations

$$
\begin{gathered}
\ddot{z}(t)+A_{0} z(t)+\frac{1}{2} C_{0}^{*} C_{0} \dot{z}(t)=C_{0}^{*} u(t), \\
y(t)=-C_{0} \dot{z}(t)+u(t)
\end{gathered}
$$

determines a well-posed linear system $\Sigma$ with input $u$ and output $y$, input and output space $U$, and state space $X=\mathcal{D}\left(A_{0}^{1 / 2}\right) \times H$. Moreover, $\Sigma$ is conservative, which means that a certain energy balance equation is satisfied both by the trajectories of $\Sigma$ and by those of its dual system. In this paper we show that $\Sigma$ is exactly controllable if and only if it is exactly observable, if and only if it is exponentially stable. Moreover, if we denote by $A$ the generator of the contraction semigroup associated with $\Sigma$ (which acts on $X$ ), then $\Sigma$ is exponentially stable if and only if one of the entries in the second column of $(i \omega I-A)^{-1}$ is uniformly bounded as a function of $\omega \in \mathbb{R}$. We also show that, under a mild assumption, $\Sigma$ is approximately controllable if and only if it is approximately observable, if and only if it is strongly stable, if and only if the dual system is strongly stable. We prove many related results and we give examples based on wave and beam equations.
\end{abstract}

Key words. well-posed linear system, exponential stability, strong stability, conservative system, exact controllability, beam equation, wave equation

AMS subject classifications. 93C25, 93B05, 93B07, 93C20, $35 \mathrm{~B} 35$

DOI. $10.1137 /$ S0363012901399295

1. Introduction and main results. This paper is a continuation of our paper [35] in which we have investigated a class of conservative linear systems with a special structure, which occur often in applications. These systems are described by a second order differential equation (in a Hilbert space) and an output equation, and they may have unbounded control and observation operators. The main aim of [35] was to prove the wellposedness, conservativity, and other regularity properties of such systems. Here we investigate conditions under which such systems are exponentially stable or strongly stable. It turns out that these stability properties are equivalent to certain controllability and observability properties as well as to certain estimates.

We recall the construction from the paper [35] in order to be able to state the new results. Let $H$ be a Hilbert space, and let $A_{0}: \mathcal{D}\left(A_{0}\right) \rightarrow H$ be a self-adjoint, positive, and boundedly invertible operator. We introduce the scale of Hilbert spaces $H_{\alpha}, \alpha \in \mathbb{R}$, as follows: for every $\alpha \geq 0, H_{\alpha}=\mathcal{D}\left(A_{0}^{\alpha}\right)$, with the norm $\|z\|_{\alpha}=\left\|A_{0}^{\alpha} z\right\|_{H}$. The space $H_{-\alpha}$ is defined by duality with respect to the pivot space $H$ as follows: $H_{-\alpha}=H_{\alpha}^{*}$ for $\alpha>0$. Equivalently, $H_{-\alpha}$ is the completion of $H$ with respect to the

* Received by the editors December 6, 2001; accepted for publication (in revised form) January 17, 2003; published electronically June 25, 2003. This research was supported by EPSRC (from the UK) under grant GR/R05048/01 and by CNRS (from France) under grant 943706 DRCI.

http://www.siam.org/journals/sicon/42-3/39929.html

${ }^{\dagger}$ Department of Mathematics, University of Nancy-I, POB 239, Vandoeuvre les Nancy 54506, France (Marius.Tucsnak@iecn.u-nancy.fr).

${ }^{\ddagger}$ Department of Electrical and Electronic Engineering, Imperial College London, Exhibition Road, London SW7 2BT, UK (G.Weiss@imperial.ac.uk). 
norm $\|z\|_{-\alpha}=\left\|A_{0}^{-\alpha} z\right\|_{H}$. The operator $A_{0}$ can be extended (or restricted) to each $H_{\alpha}$ such that it becomes a bounded operator

$$
A_{0}: H_{\alpha} \rightarrow H_{\alpha-1} \quad \forall \alpha \in \mathbb{R} .
$$

Let $C_{0}$ be a bounded linear operator from $H_{\frac{1}{2}}$ to $U$, where $U$ is another Hilbert space. We identify $U$ with its dual, so that $U=U^{*}$. We denote $B_{0}=C_{0}^{*}$ so that $B_{0} \in \mathcal{L}\left(U, H_{-\frac{1}{2}}\right)$. The class of systems studied in [35] and also here is described by

$$
\begin{gathered}
\frac{\mathrm{d}^{2}}{\mathrm{~d} t^{2}} z(t)+A_{0} z(t)+\frac{1}{2} B_{0} \frac{\mathrm{d}}{\mathrm{d} t} C_{0} z(t)=B_{0} u(t), \\
z(0)=z_{0}, \quad \dot{z}(0)=w_{0}, \\
y(t)=-\frac{\mathrm{d}}{\mathrm{d} t} C_{0} z(t)+u(t),
\end{gathered}
$$

where $t \in[0, \infty)$ is the time. The equation (1.1) is understood as an equation in $H_{-\frac{1}{2}}$, i.e., all the terms are in $H_{-\frac{1}{2}}$. Most of the linear equations modelling the damped vibrations of elastic structures can be written in the form (1.1), where $z$ stands for the displacement field and the term $B_{0} \frac{\mathrm{d}}{\mathrm{d} t} C_{0} z(t)$, informally written as $B_{0} C_{0} \dot{z}(t)$, represents a viscous feedback damping. The signal $u(t)$ is an external input with values in $U$ (often a displacement, a force, or a moment acting on the boundary), and the signal $y(t)$ is the output (measurement) with values in $U$ as well. The state $x(t)$ of this system and its state space $X$ are defined by

$$
x(t)=\left[\begin{array}{c}
z(t) \\
\dot{z}(t)
\end{array}\right], \quad X=H_{\frac{1}{2}} \times H .
$$

We will use some fairly standard notation for certain function spaces: we refer to [35, section 1] for the meaning of $\mathcal{H}^{p}(0, \infty ; W), \mathcal{H}_{l o c}^{p}(0, \infty ; W)$ (with $p \in \mathbb{N}$ ), $C^{n}(0, \infty ; W)$, and $B C^{n}(0, \infty ; W)$ (with $\left.n \in\{0,1,2, \ldots\}\right)$. We write $C$ instead of $C^{0}$.

We assume that the reader understands the concepts of a well-posed linear system and of a conservative linear system. These were explained in [35, sections $1,3,4]$ with suitable references to the literature. We will often use results from [35], which we refer to as "Part I." In such cases, we put the prefix I in front of the number of the item quoted. For example, Theorem I.1.4 refers to Theorem 1.4 in Part I, and (I.4.2) refers to formula (4.2) in Part I. The first main result of [35] has been the following (Theorem I.1.1).

THEOREM 1.1. With the above assumptions, the equations (1.1)-(1.3) determine a conservative linear system $\Sigma$ in the following sense:

There exists a conservative linear system $\Sigma$ whose input and output spaces are both $U$ and whose state space is $X$. If $u \in L^{2}([0, \infty), U)$ is the input function, $x_{0}=$ $\left[\begin{array}{l}z_{0} \\ w_{0}\end{array}\right] \in X$ is the initial state, $x=\left[\begin{array}{c}z \\ w\end{array}\right]$ is the corresponding state trajectory, and $y$ is the corresponding output function, then

(1)

$$
z \in B C\left(0, \infty ; H_{\frac{1}{2}}\right) \cap B C^{1}(0, \infty ; H) \cap \mathcal{H}_{l o c}^{2}\left(0, \infty ; H_{-\frac{1}{2}}\right) .
$$

(2) The two components of $x$ are related by $w=\dot{z}$. 
(3) $C_{0} z \in \mathcal{H}^{1}(0, \infty ; U)$ and the equations (1.1) (in $H_{-\frac{1}{2}}$ ) and (1.3) (in $U$ ) hold for almost every $t \geq 0$ (hence, $y \in L^{2}([0, \infty), U)$ ).

If $\dot{z}$ is a continuous function of $t$ with values in $H_{\frac{1}{2}}$ (see Theorems I.1.2 and I.1.4 for sufficient conditions for this to be true), then (1.1) and (1.3) can be rewritten as

$$
\begin{gathered}
\ddot{z}(t)+A_{0} z(t)+\frac{1}{2} B_{0} C_{0} \dot{z}(t)=B_{0} u(t), \\
y(t)=-C_{0} \dot{z}(t)+u(t) .
\end{gathered}
$$

We introduce the space $Z_{0}=H_{1}+A_{0}^{-1} B_{0} U$, which is a Hilbert space if we define on it a suitable norm; see Theorem I.1.2. We can rewrite the equations (1.4), (1.5) as a first order system as follows:

$$
\left\{\begin{array}{l}
\dot{x}(t)=A x(t)+B u(t) \\
y(t)=\bar{C} x(t)+u(t)
\end{array}\right.
$$

where

$$
\begin{gathered}
A=\left[\begin{array}{cc}
0 & I \\
-A_{0} & -\frac{1}{2} B_{0} C_{0}
\end{array}\right], \quad B=\left[\begin{array}{c}
0 \\
B_{0}
\end{array}\right], \\
\mathcal{D}(A)=\left\{\left[\begin{array}{c}
z \\
w
\end{array}\right] \in H_{\frac{1}{2}} \times H_{\frac{1}{2}} \mid A_{0} z+\frac{1}{2} B_{0} C_{0} w \in H\right\}, \\
\bar{C}: Z_{0} \times H_{\frac{1}{2}} \rightarrow U, \quad \bar{C}=\left[\begin{array}{ll}
0 & -C_{0}
\end{array}\right] .
\end{gathered}
$$

We denote by $C$ the restriction of $\bar{C}$ to $\mathcal{D}(A) . A$ is the generator of a strongly continuous semigroup of contractions on $X$, denoted $\mathbb{T}=\left(\mathbb{T}_{t}\right)_{t \geq 0}$. For the concepts of semigroup generator, control operator, observation operator, and transfer function of a well-posed linear system, we refer to Weiss [31, 32] or to section I.3. We denote by $\mathbb{C}_{\omega}$ the open right half-plane in $\mathbb{C}$ where $\operatorname{Re} s>\omega$. We know from Proposition I.5.3 that for any $s \in \rho(A)$ (in particular, for any $s \in \mathbb{C}_{0}$ ) the operator $s^{2} I+A_{0}+\frac{s}{2} B_{0} C_{0} \in$ $\mathcal{L}\left(H_{\frac{1}{2}}, H_{-\frac{1}{2}}\right)$ has a bounded inverse denoted $V(s)$ :

$$
V(s)=\left(s^{2} I+A_{0}+\frac{s}{2} B_{0} C_{0}\right)^{-1} \in \mathcal{L}\left(H_{-\frac{1}{2}}, H_{\frac{1}{2}}\right) .
$$

The following proposition is a restatement of a part of Theorem I.1.3.

Proposition 1.2. With the notation of Theorem 1.1 and (1.7)-(1.10), the semigroup generator of $\Sigma$ is $A$, its control operator is $B$, and its observation operator is $C$. The transfer function of $\Sigma$ is given for all $s \in \mathbb{C}_{0}$ by

$$
\mathbf{G}(s)=\bar{C}(s I-A)^{-1} B+I=I-C_{0} s V(s) B_{0},
$$

and we have $\|\mathbf{G}(s)\| \leq 1$ for all $s \in \mathbb{C}_{0}$.

Now we have all the necessary ingredients to state the new results of this paper. The following theorems use various controllability, observability, and stability concepts. The precise definition of these concepts is given in section 2 .

THEOREM 1.3. With the above notation, the following assertions are equivalent: 
(1) The pair $(A, B)$ is exactly controllable (in some finite time).

(2) The pair $(A, C)$ is exactly observable (in some finite time).

(3) The semigroup $\mathbb{T}$ is exponentially stable.

(4) The pair $(A, B)$ is optimizable.

(5) The pair $(A, C)$ is estimatable.

(6) We have $\sup _{s \in \mathbb{C}_{0}}\left\|A_{0}^{\frac{1}{2}} V(s)\right\|_{\mathcal{L}(H)}<\infty$.

(7) We have $\sup _{s \in \mathbb{C}_{0}}\|s V(s)\|_{\mathcal{L}(H)}<\infty$.

(8) For a dense subset $E$ of $\mathbb{R}$, we have $i E \subset \rho(A)$ and $\sup _{\omega \in E}\left\|A_{0}^{\frac{1}{2}} V(i \omega)\right\|_{\mathcal{L}(H)}<\infty$.

(9) For a dense subset $E$ of $\mathbb{R}$, we have $i E \subset \rho(A)$ and $\sup _{\omega \in E}\|\omega V(i \omega)\|_{\mathcal{L}(H)}<\infty$.

A more precise statement concerning the equivalence of (1), (2), and (3), which gives some information on the time of exact controllability and observability, and which is valid for any conservative system, is Proposition 3.2. The equivalence of (1)-(5) remains valid for every conservative system; see Proposition 3.3.

By a well-known theorem of Prüss and Falun, an operator semigroup $\mathbb{T}$ with generator $A$ is exponentially stable if and only if $(s I-A)^{-1}$ is uniformly bounded on $\mathbb{C}_{0}$. We refer to section 2 for precise references, further comments, and related results (Propositions 2.4 and 2.5). In the specific case of the semigroup generated by $A$ from (1.7)-(1.8), the resolvent $(s I-A)^{-1}$ can be written as a $2 \times 2$ matrix of operators; see Proposition I.5.3 (or formula (4.1) later in this paper). Thus, to verify the exponential stability of $\mathbb{T}$, we would have to verify that the four entries of this $2 \times 2$ matrix are all uniformly bounded on $\mathbb{C}_{0}$. However, conditions (6) and (7) in Theorem 1.3 tell us that, in fact, we have to verify only one of the two entries in the second column of the matrix of $(s I-A)^{-1}$. Conditions (8) and (9) tell us that, in fact, it suffices to check the boundedness of one of these entries on a dense subset of the imaginary axis, and we can still conclude exponential stability.

The version of this theorem corresponding to bounded $B$ and $C$, i.e., with $C_{0} \in$ $\mathcal{L}(H, U)$, is in Liu [22, sections 2-3] but without conditions (4)-(7). Using the boundedness of $C_{0}$ (and hence also of $B_{0}$ ), Liu was able to give in [22, Theorem 3.4] also other, Hautus-type conditions which are equivalent to the exponential stability of $\mathbb{T}$. For unbounded $C_{0}$, we were only able to obtain a Hautus-type estimate as a necessary condition for exponential stability; see Proposition 4.1.

We mention that semigroups of the type discussed in this paper do not necessarily satisfy the spectrum determined growth condition. For a counterexample (a damped wave equation on a compact manifold) see Lebeau [19].

In the proof of Theorem 1.3 (more precisely, to show that $(6) \Longrightarrow(3)$ ) we use the following proposition, which is of independent interest. For bounded $C_{0}$ this proposition follows easily from [22, Theorem 3.4], but for unbounded $C_{0}$ the proof is more delicate (see section 4). Related results for a bounded (possibly not positive) operator in place of $C_{0}^{*} C_{0}$ were given in Liu, Liu, and Rao [23].

Proposition 1.4. With the above notation, suppose that $C_{0}$ is bounded from below in the sense that there exists a $c>0$ such that $\left\|C_{0} z\right\|_{U} \geq c\|z\|_{H}$ for all $z \in H_{\frac{1}{2}}$.

Then $\mathbb{T}$ is exponentially stable.

A result similar to Theorem 1.3 holds also for strong stability, with an additional assumption on the spectrum $\sigma\left(A_{0}\right)$.

THEOREM 1.5. With the above notation, assume that $\sigma\left(A_{0}\right)$ is countable. (This happens, e.g., if $A_{0}^{-1}$ is compact.) Then the following assertions are equivalent:

(1) $\mathbb{T}$ is strongly stable.

(2) The pair $(A, C)$ is exactly observable in infinite time. 
(3) The pair $(A, C)$ is approximately observable in infinite time.

(4) $\mathbb{T}$ is weakly stable (equivalently, $\mathbb{T}^{*}$ is weakly stable).

(5) $\mathbb{T}^{*}$ is strongly stable.

(6) The pair $(A, B)$ is exactly controllable in infinite time.

(7) The pair $(A, B)$ is approximately controllable in infinite time.

(8) For any $z \in H_{1}$, if $z$ is an eigenvector of $A_{0}$, then $C_{0} z \neq 0$.

Note that the statement " $A_{0}^{-1}$ is compact" does not imply that $(s I-A)^{-1}$ is compact (to see this, take $U=H$ and $C_{0}=A_{0}^{1 / 2}$ ). This theorem follows from a more general result concerning all conservative systems; see Proposition 3.4 here. In the proof, we also use the famous strong stability theorem of Arendt and Batty [2].

Systems with $A$ and $B$ as above have been studied in Guo and Luo [10, 11], establishing connections between the exponential stability of $A$ and the exact controllability of the undamped system $\ddot{z}(t)+A_{0} z(t)=B_{0} u(t)$, under the additional hypothesis that the undamped system is well-posed. (Unfortunately, the main result on diagonal systems in [10] (Theorem 4) is incorrectly formulated, and it is also incorrectly quoted in [11].) In [11] the emphasis is on eigenvalues and eigenvectors of $A$, assuming that the eigenvalues of $A_{0}$ satisfy a gap condition and $u(t)$ is scalar.

In section 2 we give the background needed here. Section 3 concerns the stability properties of conservative systems so that the results there refer to a more general context than the main results stated earlier. In section 4 we prove our main results, while section 5 is devoted to two examples: a system involving the beam equation and another one based on the wave equation.

2. Background on controllability, observability, optimizability, estimatability, and stability. In this section we recall some controllability, observability, and stability concepts, quoting the relevant literature. Throughout this section, $U, X$, and $Y$ are Hilbert spaces and $A: \mathcal{D}(A) \rightarrow X$ is the generator of a strongly continuous semigroup $\mathbb{T}=\left(\mathbb{T}_{t}\right)_{t \geq 0}$ on $X$. The space $X_{1}$ is $\mathcal{D}(A)$ with the norm $\|z\|_{1}=\|(\beta I-$ $A) z \|$, where $\beta \in \rho(A)$ is fixed, while $X_{-1}$ is the completion of $X$ with respect to the norm $\|z\|_{-1}=\left\|(\beta I-A)^{-1} z\right\|$. We assume that the reader understands the concept of an admissible (in particular, infinite-time admissible) control operator for $\mathbb{T}$. This has been presented in section I.2 with suitable references. If $B \in \mathcal{L}\left(U, X_{-1}\right)$ is admissible, then for every $\tau \geq 0$ we denote by $\Phi_{\tau}$ the operator

$$
\Phi_{\tau} u=\int_{0}^{\tau} \mathbb{T}_{t-\sigma} B u(\sigma) \mathrm{d} \sigma
$$

as in (I.2.3). We have $\Phi_{\tau} \in \mathcal{L}\left(L^{2}([0, \infty), U), X\right)$. If $B$ is admissible, then for every $x_{0} \in X$ and every $u \in L^{2}([0, \infty), U)$, the function $x(t)=\mathbb{T}_{t} x_{0}+\Phi_{t} u$ is called the state trajectory corresponding to the initial state $x_{0}$ and the input function $u$. We have $x \in \mathcal{H}_{l o c}^{1}(0, \infty ; X)$ and $\dot{x}(t)=A x(t)+B u(t)$ (equality in $\left.X_{-1}\right)$ for almost every $t \geq 0$. If, moreover, $B$ is infinite-time admissible, then we denote, as in (I.2.5),

$$
\tilde{\Phi} u=\lim _{\tau \rightarrow \infty} \int_{0}^{\tau} \mathbb{T}_{t} B u(t) \mathrm{d} t,
$$

and we have $\tilde{\Phi} \in \mathcal{L}\left(L^{2}([0, \infty), U), X\right)$.

Similarly, we assume that the reader understands the concepts of an admissible (in particular, infinite-time admissible) observation operator for $\mathbb{T}$, also presented in section I.2. If $C \in \mathcal{L}\left(X_{1}, Y\right)$ is admissible, then we denote by $\Psi$ the unique continuous 
operator from $X$ to $L_{l o c}^{2}([0, \infty), Y)$ such that

$$
\left(\Psi x_{0}\right)(t)=C \mathbb{T}_{t} x_{0} \quad \forall x_{0} \in \mathcal{D}(A) .
$$

In particular, if $C$ is infinite-time admissible, then $\Psi \in \mathcal{L}\left(X, L^{2}([0, \infty), Y)\right)$. Recall that $B$ is an (infinite-time) admissible control operator for $\mathbb{T}$ if and only if $B^{*}$ is an (infinite-time) admissible observation operator for $\mathbb{T}^{*}$.

Definition 2.1. Let $A$ be the generator of a strongly continuous semigroup $\mathbb{T}$ on $X$, and let $B \in \mathcal{L}\left(U, X_{-1}\right)$ be an admissible control operator for $\mathbb{T}$.

The pair $(A, B)$ is exactly controllable in time $T>0$ if for every $x_{0} \in X$, there exists a $u \in L^{2}([0, T], U)$ such that $\Phi_{T} u=x_{0}$.

$(A, B)$ is exactly controllable if the above property holds for some $T>0$.

$(A, B)$ is exactly controllable in infinite time if $B$ is infinite-time admissible and the operator $\tilde{\Phi}$ from $(2.2)$ is onto.

$(A, B)$ is approximately controllable in time $T>0$ if $\operatorname{Ran} \Phi_{T}$ is dense in $X$.

$(A, B)$ is approximately controllable in infinite time if $\cup_{\tau>0} \operatorname{Ran} \Phi_{\tau}$ is dense in $X$.

$(A, B)$ is optimizable if for any $x_{0} \in X$, there exists $u \in L^{2}([0, \infty), U)$ such that the state trajectory corresponding to $x_{0}$ and $u$ is in $L^{2}([0, \infty), X)$.

Note that the exact (or approximate) controllability in infinite time of $(A, B)$ does not imply its exact (or approximate) controllability in time $T$ for some $T>0$. Clearly, exact controllability implies optimizability and also approximate controllability in some finite time. Optimizability is one possible generalization of the concept of stabilizability, as known from finite-dimensional control theory.

Remark 2.1. Let $B \in \mathcal{L}\left(U, X_{-1}\right)$ be an infinite-time admissible control operator for $\mathbb{T}$. Then $(A, B)$ is approximately controllable in infinite time if and only if the range of $\tilde{\Phi}$ from (2.2) is dense in $X$. The proof is easy.

Now we introduce the corresponding observability concepts via duality.

Definition 2.2. Suppose that $C \in \mathcal{L}\left(X_{1}, Y\right)$ is an admissible observation operator for $\mathbb{T}$. (Equivalently, $C^{*}$ is an admissible control operator for the adjoint semigroup $\mathbb{T}^{*}$.) We say that $(A, C)$ is exactly observable (in time $T$ ) (in infinite time) if $\left(A^{*}, C^{*}\right)$ is exactly controllable (in time $T$ ) (in infinite time). Similarly, $(A, C)$ is approximately observable (in time $T$ ) (in infinite time) if $\left(A^{*}, C^{*}\right)$ is approximately controllable (in time $T$ ) (in infinite time). Finally, the pair $(A, C)$ is called estimatable if $\left(A^{*}, C^{*}\right)$ is optimizable.

Let $\Psi$ be the operator defined in (2.3), and for every $\tau \geq 0$ put $\Psi_{\tau}=\mathbf{P}_{\tau} \Psi$. Then $(A, C)$ is exactly observable in time $T>0$ if and only if $\Psi_{T}$ is bounded from below. $(A, C)$ is exactly observable in infinite time if and only if $C$ is infinite-time admissible and $\Psi$ is bounded from below. $(A, C)$ is approximately observable in time $T$ (or in infinite time) if and only if $\Psi_{T} x_{0}=0$ (or $\Psi x_{0}=0$ ) implies $x_{0}=0$.

Recall that the growth bound of a strongly continuous semigroup $\mathbb{T}$ is $\omega_{0}(\mathbb{T})=$ $\lim _{t \rightarrow \infty} \frac{1}{t} \log \left\|\mathbb{T}_{t}\right\|=\inf _{t>0} \frac{1}{t} \log \left\|\mathbb{T}_{t}\right\| ;$ see, for example, Pazy [24].

Definition 2.3. The semigroup $\mathbb{T}$ is exponentially stable if its growth bound is negative: $\omega_{0}(\mathbb{T})<0 . \mathbb{T}$ is strongly stable if

$$
\lim _{t \rightarrow \infty}\left\|\mathbb{T}_{t} x_{0}\right\|=0 \quad \forall x_{0} \in X .
$$

Finally, $\mathbb{T}$ is weakly stable if $\lim _{t \rightarrow \infty}\left\langle\mathbb{T}_{t} x_{0}, y_{0}\right\rangle=0$ for all $x_{0}, y_{0} \in X$.

Let $\mathbb{T}$ be a strongly continuous semigroup on $X$ with generator $A$. A well-known spectral mapping result of Prüss [25, p. 852] implies that if the function $\left\|(s I-A)^{-1}\right\|$ is bounded on $\mathbb{C}_{0}$, then $\mathbb{T}$ is exponentially stable. A little later and independently, this 
result was explicitly stated and proved by Falun [13]. A short proof was given in Weiss $[30$, section 4]. Here we need a result which is closely related to the one just mentioned, without being an obvious consequence of it. The result is very slightly more general than another result of Falun; see [13, Theorem 3]. Moreover, the proposition below gives an estimate for the growth bound $\omega_{0}(\mathbb{T})$.

Proposition 2.4. Let $\mathbb{T}$ be a strongly continuous semigroup on $X$ with generator $A$. Assume that $\omega_{0}(\mathbb{T}) \leq 0$ and $E$ is a dense subset of $\mathbb{R}$ such that $i E \subset \rho(A)$ and

$$
\left\|(i \omega I-A)^{-1}\right\| \leq M \quad \forall \omega \in E
$$

for some $M>0$. Then $\mathbb{T}$ is exponentially stable; more precisely, $\omega_{0}(\mathbb{T}) \leq-\frac{1}{M}$.

Proof. By a result in Butzer and Berens [7, p. 31] all numbers $s \in \mathbb{C}$ with $|\operatorname{Re} s|<$ $\frac{1}{M}$ (this is a vertical strip) belong to $\rho(A)$, and we have

$$
\left\|(s I-A)^{-1}\right\| \leq \frac{M}{1-|\operatorname{Re} s| \cdot M} \quad \text { for } \quad|\operatorname{Re} s|<\frac{1}{M} .
$$

On the other hand, we know from the Hille-Yosida theorem that $\left\|(s I-A)^{-1}\right\|$ is bounded on any half-plane $\mathbb{C}_{\gamma}$ with $\gamma>0$. This fact, combined with (2.4), shows that $\left\|(s I-A)^{-1}\right\|$ is bounded on any half-plane $\mathbb{C}_{\alpha}$ with $\alpha>-\frac{1}{M}$. Now by yet another result of Falun [13, Theorem 4] we conclude that $\omega_{0}(\mathbb{T}) \leq-\frac{1}{M}$. (The last step is equivalent to applying the Prüss-Huang result mentioned before the proposition for the semigroups generated by $A+\lambda I$ with $\lambda<\frac{1}{M}$.)

Proposition 2.5. Suppose that $X, \mathbb{T}, A, U$, and $B$ are as in Definition 2.1. Then the following three statements are equivalent:

(1) $\mathbb{T}$ is exponentially stable.

(2) $(A, B)$ is optimizable, $\mathbb{C}_{0} \subset \rho(A)$, and for some $M>0$

$$
\left\|(s I-A)^{-1} B\right\|_{\mathcal{L}(U, X)} \leq M \quad \forall s \in \mathbb{C}_{0} .
$$

(3) $(A, B)$ is optimizable, $\omega_{0}(\mathbb{T}) \leq 0$, there exists a dense subset of $\mathbb{R}$, denoted $E$, such that $i E \subset \rho(A)$, and for some $M>0$

$$
\left\|(i \omega I-A)^{-1} B\right\|_{\mathcal{L}(U, X)} \leq M \quad \forall \omega \in E .
$$

Proof. The equivalence of (1) and (2) is exactly Proposition 5.1 in Weiss and Rebarber [33]. It is easy to see that (1) implies (3) with $E=\mathbb{R}$ (by also using (2) and limits as $s \rightarrow i \omega$ ). Now suppose that (3) holds (this implies $\mathbb{C}_{0} \subset \rho(A)$ ). We argue as in the proof of [33, Proposition 5.1], obtaining formula (5.1) from [33] valid for all $s \in \mathbb{C}_{0}$. Taking limits, we see that this formula holds also with $i \omega$ in place of $s \in \mathbb{C}_{0}$, where $\omega \in E$. Continuing to reason as in [33], we obtain that $(i \omega I-A)^{-1}$ is uniformly bounded as a function of $\omega \in E$. Since $\omega_{0}(\mathbb{T}) \leq 0$, we can apply Proposition 2.4 to conclude that $\mathbb{T}$ is exponentially stable.

Observability (or dually, controllability) and strong stability concepts are linked to properties of Lyapunov equations, and we state in dual form the following result from section 3 of Hansen and Weiss [12].

Proposition 2.6. Let $A$ be the generator of the strongly continuous semigroup $\mathbb{T}$ on $X$, and let $C \in \mathcal{L}\left(X_{1}, Y\right)$ be an admissible observation operator for $\mathbb{T}$. Then the following three statements are equivalent:

(a) There exist operators $\Pi \in \mathcal{L}(X), \Pi \geq 0$, which satisfy the following equation:

$$
A^{*} \Pi z+\Pi A z=-C^{*} C z \quad \forall z \in \mathcal{D}(A) .
$$


(b) $C$ is an infinite-time admissible observation operator for $\mathbb{T}$.

(c) There exists an operator $P \in \mathcal{L}(X)$ such that for any $z \in \mathcal{D}(A)$

$$
P z=\lim _{\tau \rightarrow \infty} \int_{0}^{\tau} \mathbb{T}_{t}^{*} C^{*} C \mathbb{T}_{t} z \mathrm{~d} t .
$$

Moreover, if $C$ is infinite-time admissible, then the following statements hold:

(d) $P$ from (2.6) is the smallest nonnegative solution of (2.5).

(e) If $P$ is invertible, then $\mathbb{T}$ is strongly stable.

(f) If $\mathbb{T}$ is strongly stable, then $P$ is the unique self-adjoint solution of (2.5).

(g) If $\mathbb{T}$ is uniformly bounded and $P>0$, then $\mathbb{T}$ is weakly stable.

The operator $P$ introduced above is called the observability Gramian of $(A, C)$, and (2.5) is called a Lyapunov equation. Note that, in terms of the operator $\Psi$ from (2.3), we have $P=\Psi^{*} \Psi$. The following is well known and easy to prove.

Proposition 2.7. Suppose that $C$ is an infinite-time admissible observation operator for the semigroup $\mathbb{T}$ generated by $A$. Then $(A, C)$ is approximately observable in infinite time if and only if $P>0$ (where $P$ is the observability Gramian of $(A, C)$ ).

The controllability Gramian of $(A, B)$ is, by definition, the observability Gramian of $\left(A^{*}, B^{*}\right)$. Thus, the controllability Gramian of $(A, B)$ is defined by

$$
R x=\lim _{\tau \rightarrow \infty} \int_{0}^{\tau} \mathbb{T}_{t} B B^{*} \mathbb{T}_{t}^{*} x \mathrm{~d} t \quad \forall x \in \mathcal{D}\left(A^{*}\right),
$$

we have $R=\tilde{\Phi} \tilde{\Phi}^{*}$, and the Lyapunov equation satisfied by $R$ is

$$
R A^{*} z+A R z=-B B^{*} z \quad \forall z \in \mathcal{D}\left(A^{*}\right) .
$$

The dual version of Proposition 2.6 is straightforward.

For more details on Gramians we refer to Hansen and Weiss [12], Jacob and Partington [14], Russell and Weiss [28], and Grabowski [8]. For more details on exact controllability in an operator-theoretic setting we also refer to Avdonin and Ivanov [3], Jacob and Zwart [15], Rebarber and Weiss [27], Tucsnak and Weiss [29], and the references therein. In the PDE setting, the relevant literature is overwhelming, and we mention the books of Lions [20], Lagnese and Lions [17], Bensoussan et al. [6], Komornik [16], and the paper of Bardos, Lebeau, and Rauch [5].

3. Conservative linear systems. Recall from section I.3 that for any wellposed system $\Sigma$ with input function $u$, state trajectory $x$, and output function $y$,

$$
\left[\begin{array}{l}
x(\tau) \\
\mathbf{P}_{\tau} y
\end{array}\right]=\Sigma_{\tau}\left[\begin{array}{l}
x(0) \\
\mathbf{P}_{\tau} u
\end{array}\right]
$$

where $\mathbf{P}_{\tau}$ denotes the truncation of a function to $[0, \tau]$ and

$$
\Sigma_{\tau}=\left[\begin{array}{ll}
\mathbb{T}_{\tau} & \Phi_{\tau} \\
\Psi_{\tau} & \mathbb{F}_{\tau}
\end{array}\right]
$$

We denote the input, state, and output spaces of $\Sigma$ by $U, X$, and $Y$, respectively. Then the operators $\Sigma_{\tau}$ appearing above are bounded from $X \times L^{2}([0, \tau], U)$ to $X \times$ $L^{2}([0, \tau], Y)$, which means that for some $c_{\tau} \geq 0$

$$
\|x(\tau)\|^{2}+\int_{0}^{\tau}\|y(t)\|^{2} \mathrm{~d} t \leq c_{\tau}^{2}\left(\|x(0)\|^{2}+\int_{0}^{\tau}\|u(t)\|^{2} \mathrm{~d} t\right) .
$$


As explained in section I.1, the system $\Sigma$ is conservative if the operators $\Sigma_{\tau}$ are unitary from $X \times L^{2}([0, \tau], U)$ to $X \times L^{2}([0, \tau], Y)$. This implies that for any input function $u \in \mathcal{H}^{1}(0, \infty ; U)$ and any initial state $x(0)=x_{0} \in X$ with $A x_{0}+B u(0) \in X$, the function $\|x(t)\|^{2}$ is in $C^{1}[0, \infty)$ and

$$
\frac{\mathrm{d}}{\mathrm{d} t}\|x(t)\|^{2}=\|u(t)\|^{2}-\|y(t)\|^{2} \quad \forall t \geq 0 ;
$$

see Proposition I.4.3. Conversely, if (3.3) holds for both the system $\Sigma$ and for its dual system $\Sigma^{d}$, then $\Sigma$ is conservative; see Corollary I.4.4.

Proposition 3.1. Let $\Sigma$ be a conservative linear system with input space $U$, state space $X$, output space $Y$, semigroup $\mathbb{T}$, control operator $B$, observation operator $C$, and transfer function $\mathbf{G}$. Then the following statements are true:

(1) $\mathbb{T}$ is a semigroup of contractions.

(2) $B$ is infinite-time admissible.

(3) $C$ is infinite-time admissible.

(4) $\|\mathbf{G}(s)\| \leq 1$ for all $s \in \mathbb{C}_{0}$.

Indeed, the above proposition is an immediate consequence of Proposition I.4.5 (which concerns the larger class of dissipative linear systems). The following proposition is probably well known (especially the equivalence of (2) and (3)), but we are not aware of a reference which states the equivalence of all three conditions.

Proposition 3.2. With the notation of Proposition 3.1 and denoting the generator of $\mathbb{T}$ by $A$ for each $\tau>0$, the following statements are equivalent:

(1) The pair $(A, B)$ is exactly controllable in time $\tau$.

(2) The pair $(A, C)$ is exactly observable in time $\tau$.

(3) $\left\|\mathbb{T}_{\tau}\right\|<1$ (in particular, $\mathbb{T}$ is exponentially stable).

Proof. (3) $\Longrightarrow(2)$ With the notation from (3.2), it is clear that for all $x_{0} \in X$

$$
\left\|\mathbb{T}_{\tau} x_{0}\right\|^{2}+\left\|\Psi_{\tau} x_{0}\right\|^{2}=\left\|x_{0}\right\|^{2} \quad \forall \tau \geq 0 .
$$

If (3) holds, then $\left\|\mathbb{T}_{\tau}\right\|^{2}=1-\varepsilon^{2}$ with $\varepsilon>0$. Now (3.4) implies that $\left\|\Psi_{\tau} x_{0}\right\|^{2} \geq$ $\varepsilon^{2}\left\|x_{0}\right\|^{2}$, so that $\Sigma$ is exactly observable in time $\tau$.

$(2) \Longrightarrow(3)$ If (2) holds, then there exists $\varepsilon>0$ such that $\left\|\Psi_{\tau} x_{0}\right\| \geq \varepsilon\left\|x_{0}\right\|$ for all $x_{0} \in X$. Now (3.4) implies that $\left\|\mathbb{T}_{\tau} x_{0}\right\|^{2} \leq\left(1-\varepsilon^{2}\right)\left\|x_{0}\right\|^{2}$; hence $\left\|\mathbb{T}_{\tau}\right\|<1$.

(3) $\Longleftrightarrow$ (1) (3) is equivalent to the fact that $\left\|\mathbb{T}_{\tau}^{*}\right\|<1$. (1) is equivalent to the fact that $\left(A^{*}, B^{*}\right)$ is exactly observable in time $\tau$. Since the dual system $\Sigma^{d}$ is conservative, according to Proposition I.4.2 and the equivalence of (2) and (3) proved earlier, we get that (1) is equivalent to (3).

The equivalence of (1)-(5) in Theorem 1.3 is an immediate consequence of Theorem 1.1, Proposition 1.2, and the following simple result about conservative systems.

Proposition 3.3. With the notation of Proposition 3.2, the following five statements are equivalent:

(1) The pair $(A, B)$ is exactly controllable.

(2) The pair $(A, C)$ is exactly observable.

(3) $\mathbb{T}$ is exponentially stable.

(4) The pair $(A, B)$ is optimizable.

(5) The pair $(A, C)$ is estimatable.

Proof. The equivalence of (1)-(3) follows from the previous proposition. It is well known and easy to see that (1) implies (4) and (2) implies (5) (for any well-posed system). Suppose that (5) holds. We know from Proposition 3.1 that $C$ is infinitetime admissible. Now it follows from [33, Proposition 5.5] that (3) holds. The proof of $(4) \Longrightarrow(3)$ is similar, by using the dual version of [33, Proposition 5.5]. 
Another result linking strong stability, observability, and controllability of conservative systems is the following. It is related to Proposition 6 in [34].

Proposition 3.4. Let $\Sigma, \mathbb{T}, A, B$, and $C$ be as in Proposition 3.2. Assume that the intersection $\sigma(A) \cap i \mathbb{R}$ is countable. (This happens, for example, if $(\beta I-A)^{-1}$ is compact for some $\beta \in \rho(A)$.) Then the following seven assertions are equivalent:

(1) $\mathbb{T}$ is strongly stable.

(2) The pair $(A, C)$ is exactly observable in infinite time.

(3) The pair $(A, C)$ is approximately observable in infinite time.

(4) $\mathbb{T}$ is weakly stable (equivalently, $\mathbb{T}^{*}$ is weakly stable).

(5) $\mathbb{T}^{*}$ is strongly stable.

(6) The pair $(A, B)$ is exactly controllable in infinite time.

(7) The pair $(A, B)$ is approximately controllable in infinite time.

Proof. (1) $\Longrightarrow(2)$ If $\mathbb{T}$ is strongly stable, then we see from (3.4) that $\lim _{\tau \rightarrow \infty}\left\|\Psi_{\tau} x_{0}\right\|=\left\|x_{0}\right\|$. This implies that $\Psi$ is an isometry from $X$ to $L^{2}([0, \infty), Y)$.

$(2) \Longrightarrow(3)$ This implication is obvious.

$(3) \Longrightarrow(4)$ The fact that $\Sigma$ is conservative implies that $C$ is infinite-time admissible. According to Proposition 2.7, (3) means that $P>0$, where $P$ is the observability Gramian. By the last part of Proposition 2.6, $\mathbb{T}$ is weakly stable.

$(4) \Longrightarrow(1)$ Since $\mathbb{T}$ is weakly stable, $A$ has no eigenvalues on $i \mathbb{R}$. Together with the assumption that $\sigma(A) \cap i \mathbb{R}$ is countable, this means that the conditions of the famous stability theorem of Arendt and Batty [2] are satisfied. According to this theorem, $\mathbb{T}$ is strongly stable.

$(4) \Longleftrightarrow(5) \Longleftrightarrow(6) \Longleftrightarrow(7)$ This is similar to the equivalence of (1)-(4) but with the dual system $\Sigma^{d}$ in place of $\Sigma$. (Recall that $\Sigma^{d}$ is also conservative.)

4. Proof of the main results. In this section we prove Theorems 1.3, 1.4, 1.5 as well as other related results. We use the assumptions and the notation from section 1: The conservative linear system $\Sigma$ is the one constructed in Theorem 1.1 from the operators $A_{0} \in \mathcal{L}\left(H_{1}, H\right)$ and $C_{0} \in \mathcal{L}\left(H_{\frac{1}{2}}, U\right)$. The spaces $H_{\alpha}$ with $\alpha \in \mathbb{R}$ are constructed from the fractional powers of $A_{0}$. The notation $\|z\|_{\alpha}$ means the norm of $z$ in $H_{\alpha}$; in particular, $\|z\|_{0}$ is the norm of $z$ in $H$. We put $B_{0}=C_{0}^{*}$. The operators $A$ and $B$ are defined in (1.7), (1.8) and $\bar{C}$ is defined in (1.9). $C$ is the restriction of $\bar{C}$ to $\mathcal{D}(A)$. The semigroup of contractions generated by $A$ on $X=H_{\frac{1}{2}} \times H$ is denoted by $\mathbb{T}$, and the transfer function of $\Sigma$ is denoted by G. Recall also the $\mathcal{L}\left(H_{-\frac{1}{2}}, H_{\frac{1}{2}}\right)$-valued function $V(s)$ from (1.10) and the space $Z_{0}$ defined after (1.5).

Proposition 4.1. With the above notation, if $\mathbb{T}$ is exponentially stable, then denoting $M=\sup _{\omega \in \mathbb{R}}\left\|(i \omega I-A)^{-1}\right\|_{\mathcal{L}(X)}$ we have for every $z \in H_{\frac{1}{2}}$

$$
\left\|\left(\omega^{2} I-A_{0}\right) z\right\|_{-\frac{1}{2}}+\frac{\omega}{2}\left\|B_{0} C_{0} z\right\|_{-\frac{1}{2}} \geq \frac{1}{M}\|z\|_{0} \quad \forall \omega \in[0, \infty) .
$$

Proof. We know from Proposition I.5.3 that on $H_{\frac{1}{2}} \times H_{-\frac{1}{2}}$ (in particular, on $X$ ) we have for all $s \in \rho(A)$ with $s \neq 0$

$$
(s I-A)^{-1}=\left[\begin{array}{cc}
\frac{1}{s}\left[I-V(s) A_{0}\right] & V(s) \\
-V(s) A_{0} & s V(s)
\end{array}\right] .
$$

For $s=0$ the formula remains valid if we replace the left upper block in the matrix with $\frac{1}{2} A_{0}^{-1} B_{0} C_{0}$; see (I.5.4). Since $\mathbb{T}$ is exponentially stable, any point $i \omega$ (with 
$\omega \in \mathbb{R})$ is in $\rho(A)$ and $(i \omega I-A)^{-1}$ is uniformly bounded. Looking at the left lower block of $(i \omega I-A)^{-1}$, we have

$$
\sup _{\omega \in \mathbb{R}}\left\|V(i \omega) A_{0}^{\frac{1}{2}}\right\|_{\mathcal{L}(H)}=\sup _{\omega \in \mathbb{R}}\left\|V(i \omega) A_{0}\right\|_{\mathcal{L}\left(H_{\frac{1}{2}}, H\right)} \leq \sup _{\omega \in \mathbb{R}}\left\|(i \omega I-A)^{-1}\right\|_{\mathcal{L}(X)}=M .
$$

The last estimate means that for any $g \in H$ and any $\omega \in \mathbb{R}$,

$$
\left\|V(i \omega) A_{0}^{\frac{1}{2}} g\right\|_{0} \leq M\|g\|_{0} .
$$

If we choose $g=A_{0}^{-\frac{1}{2}}\left(-\omega^{2} I+A_{0}+\frac{i \omega}{2} B_{0} C_{0}\right) z$ with $z \in H_{\frac{1}{2}}$ fixed, then we get that for all $\omega \in \mathbb{R}$

$$
M\left\|A_{0}^{-\frac{1}{2}}\left(-\omega^{2} I+A_{0}+\frac{i \omega}{2} B_{0} C_{0}\right) z\right\|_{0} \geq\|z\|_{0} .
$$

From here, using the triangle inequality, we get the estimate in the proposition.

Proof of Proposition 1.4. Recall that the inner product on $X$ is defined by

$$
\left\langle\left[\begin{array}{c}
z_{1} \\
w_{1}
\end{array}\right],\left[\begin{array}{c}
z_{2} \\
w_{2}
\end{array}\right]\right\rangle_{X}=\left\langle A_{0}^{\frac{1}{2}} z_{1}, A_{0}^{\frac{1}{2}} z_{2}\right\rangle_{H}+\left\langle w_{1}, w_{2}\right\rangle_{H}
$$

In what follows, we drop the subscript $H$ when writing the inner product on $H$ (but we use subscripts for other spaces). We define a new inner product on $X$ by

$$
\begin{aligned}
\left\langle\left[\begin{array}{c}
z_{1} \\
w_{1}
\end{array}\right],\left[\begin{array}{c}
z_{2} \\
w_{2}
\end{array}\right]\right\rangle_{\text {new }} & =\left\langle A_{0}^{\frac{1}{2}} z_{1}, A_{0}^{\frac{1}{2}} z_{2}\right\rangle+\delta\left\langle w_{1}, z_{2}\right\rangle+\delta\left\langle z_{1}, w_{2}\right\rangle+\left\langle w_{1}, w_{2}\right\rangle \\
& =\left\langle\left[\begin{array}{cc}
I & \delta A_{0}^{-1} \\
\delta I & I
\end{array}\right]\left[\begin{array}{c}
z_{1} \\
w_{1}
\end{array}\right],\left[\begin{array}{c}
z_{2} \\
w_{2}
\end{array}\right]\right\rangle_{X},
\end{aligned}
$$

where $\delta>0$ is such that $\delta\left\|A_{0}^{-\frac{1}{2}}\right\|<1$. (Later we shall impose further restrictions on $\delta$.) The $2 \times 2$ matrix $J$ appearing above defines a self-adjoint and positive bounded operator on $X$. Indeed, $J \geq 0$ follows from the Cauchy-Schwarz inequality:

$$
\begin{aligned}
\left\langle\left[\begin{array}{cc}
I & \delta A_{0}^{-1} \\
\delta I & I
\end{array}\right]\left[\begin{array}{c}
z \\
w
\end{array}\right],\left[\begin{array}{c}
z \\
w
\end{array}\right]\right\rangle_{X} & \geq\left\|A_{0}^{\frac{1}{2}} z\right\|^{2}-2 \delta\|z\| \cdot\|w\|+\|w\|^{2} \\
& \geq\left\|A_{0}^{\frac{1}{2}} z\right\|^{2}-2 \delta\left\|A_{0}^{-\frac{1}{2}}\right\| \cdot\left\|A_{0}^{\frac{1}{2}} z\right\| \cdot\|w\|+\|w\|^{2} \\
& \geq\left\|A_{0}^{\frac{1}{2}} z\right\|^{2}-2\left\|A_{0}^{\frac{1}{2}} z\right\| \cdot\|w\|+\|w\|^{2} \\
& =\left(\left\|A_{0}^{\frac{1}{2}} z\right\|-\|w\|\right)^{2} \geq 0 .
\end{aligned}
$$

It is easy to check that $J$ is boundedly invertible, hence $J>0$, which shows that our definition of a new inner product is correct, and the new norm on $X$ defined by $\|x\|_{\text {new }}=\sqrt{\langle x, x\rangle}_{\text {new }}$ is equivalent to the original norm. Thus, it will suffice to prove that $\mathbb{T}$ is exponentially stable with respect to the new norm.

We shall estimate $\langle A x, x\rangle_{\text {new }}$, where $x=\left[\begin{array}{c}z \\ w\end{array}\right] \in \mathcal{D}(A)$. We have

$$
\operatorname{Re}\langle A x, x\rangle_{\text {new }}=\operatorname{Re}\langle(J-I) A x, x\rangle_{X}+\operatorname{Re}\langle A x, x\rangle_{X} .
$$


We know from (I.5.3) that $\operatorname{Re}\langle A x, x\rangle_{X}=-\frac{1}{2}\left\|C_{0} w\right\|_{U}^{2}$. Computing the product $(J-$ I) $A$, we get that

$$
\operatorname{Re}\langle A x, x\rangle_{\text {new }}=-\delta\left\|A_{0}^{\frac{1}{2}} z\right\|^{2}-\frac{\delta}{2} \operatorname{Re}\left\langle C_{0} w, C_{0} z\right\rangle_{U}+\delta\|w\|^{2}-\frac{1}{2}\left\|C_{0} w\right\|_{U}^{2} .
$$

Now remember that $C_{0}$ is bounded from below, so that $\|w\| \leq \frac{1}{c}\left\|C_{0} w\right\|_{U}$. Therefore, the above estimate and the Cauchy-Schwarz inequality imply

$$
\operatorname{Re}\langle A x, x\rangle_{\text {new }} \leq-\delta\left\|A_{0}^{\frac{1}{2}} z\right\|^{2}+\frac{\delta}{2}\left\|C_{0} w\right\|_{U} \cdot\left\|C_{0} z\right\|_{U}-\left(\frac{1}{2}-\frac{\delta}{c^{2}}\right)\left\|C_{0} w\right\|_{U}^{2} .
$$

Let $k>0$ be such that $\left\|C_{0} z\right\|_{U} \leq k\left\|A_{0}^{\frac{1}{2}} z\right\|$ for all $z \in H_{\frac{1}{2}}$. Then

$$
\operatorname{Re}\langle A x, x\rangle_{\text {new }} \leq-\delta\left\|A_{0}^{\frac{1}{2}} z\right\|^{2}+\frac{\delta k}{2}\left\|C_{0} w\right\|_{U} \cdot\left\|A_{0}^{\frac{1}{2}} z\right\|-\left(\frac{1}{2}-\frac{\delta}{c^{2}}\right)\left\|C_{0} w\right\|_{U}^{2} .
$$

The right-hand side above is a quadratic form in the two numbers $\left\|A_{0}^{\frac{1}{2}} z\right\|$ and $\left\|C_{0} w\right\|_{U}$. The matrix of this quadratic form is

$$
Q=-\left[\begin{array}{cc}
\delta & -\frac{\delta k}{4} \\
-\frac{\delta k}{4} & \frac{1}{2}-\frac{\delta}{c^{2}}
\end{array}\right] .
$$

This $Q$ will be negative definite if

$$
\frac{1}{2}-\frac{\delta}{c^{2}}>0 \quad \text { and } \quad 16\left(\frac{1}{2}-\frac{\delta}{c^{2}}\right)>\delta k^{2}
$$

Both of these conditions can be satisfied if we choose $\delta$ sufficiently small. Suppose that $\delta$ has been correctly chosen so that $Q \leq-\gamma I$ for some $\gamma>0$. Then we obtain

$$
\begin{aligned}
\operatorname{Re}\langle A x, x\rangle_{\text {new }} & \leq-\gamma\left(\left\|A_{0}^{\frac{1}{2}} z\right\|^{2}+\left\|C_{0} w\right\|_{U}^{2}\right) \\
& \leq-\gamma\left(\left\|A_{0}^{\frac{1}{2}} z\right\|^{2}+c^{2}\|w\|^{2}\right) \leq-\gamma \min \left(1, c^{2}\right)\|x\|_{X}^{2} .
\end{aligned}
$$

Recall that the two norms on $X$ are equivalent so that $\|x\|_{X} \geq m\|x\|_{\text {new }}$ for some $m>0$. Denoting $\eta=\gamma \min \left(1, c^{2}\right) m^{2}$ (so that $\eta>0$ ), we obtain

$$
\operatorname{Re}\langle A x, x\rangle_{\text {new }} \leq-\eta\|x\|_{\text {new }}^{2}
$$

so that $A+\eta I$ is dissipative with respect to the new inner product. Hence, the growth bound of $\mathbb{T}$ (which does not depend on the norm) is $\omega_{0}(\mathbb{T}) \leq-\eta$.

Lemma 4.2. If we define $B_{b}=\left[\begin{array}{l}0 \\ I\end{array}\right] \in \mathcal{L}(H, X)$, then $\left(A, B_{b}\right)$ is optimizable.

Proof. Consider a new conservative linear system $\tilde{\Sigma}$ obtained from the same operator $A_{0}$ on the same Hilbert space $H$ but with a larger input space $\tilde{U}$ and with $C_{0}$ replaced by $\tilde{C}_{0}$, which are defined as follows:

$$
\tilde{U}=U \times H, \quad \tilde{C}_{0}=\left[\begin{array}{c}
C_{0} \\
I
\end{array}\right] .
$$


Thus, following the standard construction from section $1, B_{0}$ will be replaced by $\tilde{B}_{0}=\tilde{C}_{0}^{*}=\left[B_{0} I\right]$. According to (1.7), the semigroup $\tilde{\mathbb{T}}$ of $\tilde{\Sigma}$ is generated by

$$
\tilde{A}=\left[\begin{array}{cc}
0 & I \\
-A_{0} & -\frac{1}{2} B_{0} C_{0}-\frac{1}{2} I
\end{array}\right]
$$

with $\underset{\mathcal{D}}{\mathcal{A}}(\tilde{A})=\mathcal{D}(A)$ as defined in (1.8). It is clear that $\tilde{C}_{0}$ is bounded from below, so that $\tilde{\mathbb{T}}$ is exponentially stable according to Proposition 1.4.

Now consider the system $\Sigma_{b}$ with input space $H$, state space $X$, and output space $H$ described by

$$
\left\{\begin{array}{l}
\dot{x}(t)=A x(t)+B_{b} u(t) \\
y(t)=B_{b}^{*} x(t)
\end{array}\right.
$$

Clearly $\Sigma_{b}$ is well-posed, since $B_{b}$ is bounded. The static output feedback $u=-\frac{1}{2} y$ applied to this system leads to a closed-loop system whose semigroup generator is $A-\frac{1}{2} B_{b}^{*} B_{b}=\tilde{A}$, which (as we already know) is exponentially stable. In particular, it follows that for any initial state $x_{0} \in X$, the functions $u$ and $x$ defined by $u(t)=$ $-\frac{1}{2} B_{b}^{*} \tilde{\mathbb{T}}_{t} x_{0}$ and $x(t)=\tilde{\mathbb{T}}_{t} x_{0}$ are both in $L^{2}$. Thus, $\left(A, B_{b}\right)$ is optimizable.

Proof of Theorem 1.3. According to Theorem 1.1 and Proposition 1.2, $\Sigma$ is a conservative linear system with semigroup generator $A$, control operator $B$, and observation operator $C$. Now the equivalence of (1)-(5) in Theorem 1.3 follows from Proposition 3.3. It is also easy to see that (3) implies (6), (7), (8), and (9). Indeed, if $\mathbb{T}$ is exponentially stable, then $(s I-A)^{-1}$ exists and is uniformly bounded on $\mathbb{C}_{\alpha}$ for some $\alpha<0$; see the proof of Proposition 2.4. Looking at the right column of $(s I-A)^{-1}$ in (4.1), we obtain that (6)-(9) all hold.

We prove the equivalence of (6) and (7). Suppose that (7) is false; i.e., there is a sequence $\left(s_{n}\right)$ in $\mathbb{C}_{0}$ such that $\left\|s_{n} V\left(s_{n}\right)\right\| \rightarrow \infty$. Since $\mathbb{T}$ is uniformly bounded, $\left\|(s I-A)^{-1}\right\|$ is bounded on any right half-plane $\mathbb{C}_{\gamma}$ with $\gamma>0$. Since $s V(s)$ is one of the entries of $(s I-A)^{-1}$, it follows that for large $n$ the sequence $\left(s_{n}\right)$ must be outside $\mathbb{C}_{\gamma}$. Since this is true for each $\gamma>0$, we must have $\operatorname{Re} s_{n} \rightarrow 0$. Since $0 \in \rho(A)$, $\|s V(s)\|$ is bounded on a neighborhood of 0 . Thus, without loss of generality we may assume that $\left|\operatorname{Im} s_{n}\right| \geq \varepsilon>0$.

By the uniform boundedness theorem, there exists a vector $x \in H$ such that

$$
\lambda_{n}=\left\|s_{n} V\left(s_{n}\right) x\right\| \rightarrow \infty .
$$

Denote $z_{n}=\frac{1}{\lambda_{n}} V\left(s_{n}\right) x$; then clearly $z_{n} \in H_{\frac{1}{2}},\left\|s_{n} z_{n}\right\|=1$ (hence $\left\|z_{n}\right\|$ is bounded), and

$$
\frac{1}{\lambda_{n}} x=s_{n}^{2} z_{n}+A_{0} z_{n}+\frac{s_{n}}{2} B_{0} C_{0} z_{n} \rightarrow 0 \text { in } H .
$$

Taking inner products with $z_{n}$, we obtain

$$
\frac{1}{\lambda_{n}}\left\langle x, z_{n}\right\rangle=s_{n}^{2}\left\|z_{n}\right\|^{2}+\left\|A_{0}^{\frac{1}{2}} z_{n}\right\|^{2}+\frac{s_{n}}{2}\left\|C_{0} z_{n}\right\|^{2} \rightarrow 0 .
$$

Looking here only at the imaginary parts and dividing by $\operatorname{Im} s_{n}$, we obtain

$$
2\left(\operatorname{Re} s_{n}\right)\left\|z_{n}\right\|^{2}+\frac{1}{2}\left\|C_{0} z_{n}\right\|^{2} \rightarrow 0 ;
$$


in particular, $\left\|C_{0} z_{n}\right\| \rightarrow 0$. Now we look at the real parts of the terms in (4.2):

$$
-\left|s_{n}\right|^{2}\left\|z_{n}\right\|^{2}+2\left(\operatorname{Re} s_{n}\right)^{2}\left\|z_{n}\right\|^{2}+\left\|A_{0}^{\frac{1}{2}} z_{n}\right\|^{2}+\frac{\operatorname{Re} s_{n}}{2}\left\|C_{0} z_{n}\right\|^{2} \rightarrow 0 .
$$

Recalling that $\operatorname{Re} s_{n} \rightarrow 0$ and $\left\|s_{n} z_{n}\right\|=1$, we conclude that $\lim _{n \rightarrow \infty}\left\|A_{0}^{\frac{1}{2}} z_{n}\right\|=1$. Thus, $\lim _{n \rightarrow \infty} \frac{1}{\lambda_{n}}\left\|A_{0}^{\frac{1}{2}} V\left(s_{n}\right) x\right\|=1$ so that $\left\|A_{0}^{\frac{1}{2}} V\left(s_{n}\right)\right\| \rightarrow \infty$. We have obtained that assertion (6) is false, so (6) implies (7). The proof of the fact that (7) implies (6) is similar with the following modifications: $x$ and $\lambda_{n}$ are now chosen such that $\lambda_{n}=$ $\left\|A_{0}^{\frac{1}{2}} V\left(s_{n}\right) x\right\| \rightarrow \infty$. We take again $z_{n}=\frac{1}{\lambda_{n}} V\left(s_{n}\right) x$, and now $\left\|A_{0}^{\frac{1}{2}} z_{n}\right\|=1$ (instead of $\left.\left\|s_{n} z_{n}\right\|=1\right)$. Now the reasoning up to (4.4) remains the same, and from (4.4) we conclude that $\lim _{n \rightarrow \infty}\left\|s_{n} z_{n}\right\|=1$, which implies that $\left\|s_{n} V\left(s_{n}\right)\right\| \rightarrow \infty$.

To prove the equivalence of (8) and (9), we argue similarly as in the proof of the equivalence of (6) and (7), but now $\operatorname{Re} s_{n}=0$. This makes the proof simpler, since now we do not need (4.3) and in (4.4) two terms disappear.

We prove that (6) implies (3). If (6) (and hence also (7)) holds, then we see from (4.1) that $(s I-A)^{-1} B_{b}$ is uniformly bounded on $\mathbb{C}_{0}$, where $B_{b}$ is the operator from Lemma 4.2. Since, by the same lemma, $\left(A, B_{b}\right)$ is optimizable, we can apply Proposition 2.5 (the equivalence of points (1) and (2) in that proposition) to conclude that $\mathbb{T}$ is exponentially stable, i.e., (3) holds.

Finally, we prove that (8) implies (3). If (8) (and hence also (9)) holds, then we see from (4.1) that $(i \omega I-A)^{-1} B_{b}$ is uniformly bounded for $\omega \in E$, where $B_{b}$ is the operator from Lemma 4.2. Since $\left(A, B_{b}\right)$ is optimizable, and since $\mathbb{T}$ is uniformly bounded, the conditions in point (3) of Proposition 2.5 are satisfied. According to Proposition $2.5, \mathbb{T}$ is exponentially stable.

In order to prove Theorem 1.5, we have to prove several preliminary results. We denote by $\sigma_{p}(A)$ the set of eigenvalues (the point spectrum) of $A$.

Lemma 4.3. If $\lambda \in \sigma_{p}(A)$ and $x \in \mathcal{D}(A)$ is a corresponding eigenvector (i.e., $(\lambda I-A) x=0$ and $x \neq 0)$, then $x$ is of the form

$$
x=\left[\begin{array}{c}
z \\
\lambda z
\end{array}\right], \quad z \in Z_{0},
$$

where $Z_{0}=H_{1}+A_{0}^{-1} B_{0} U$ and

$$
\left(\lambda^{2} I+A_{0}+\frac{\lambda}{2} B_{0} C_{0}\right) z=0 .
$$

If $\lambda \notin \mathbb{R}$, then this implies

$$
\left\|C_{0} z\right\|^{2}=4|\operatorname{Re} \lambda| \cdot\|z\|^{2}, \quad\left\langle A_{0} z, z\right\rangle=|\lambda|^{2} \cdot\|z\|^{2} .
$$

Proof. The formulas (4.5) and (4.6) are an immediate consequence of $\mathcal{D}(A) \subset$ $Z_{0} \times H_{\frac{1}{2}}$ (which follows from (1.8)) and of $(\lambda I-A) x=0$. If we take the scalar product of the sides of (4.6) with $z$ and use the extension of the scalar product to the duality pairing between $H_{-\frac{1}{2}}$ and $H_{\frac{1}{2}}$, we obtain

$$
\left\langle\left(\lambda^{2} I+A_{0}\right) z, z\right\rangle+\frac{\lambda}{2}\left\|C_{0} z\right\|^{2}=0 .
$$

Since $\mathbb{T}$ is a contraction semigroup, $\lambda$ must be in the closed left half-plane. Denoting $\lambda=-\sigma+i \omega$ with $\sigma \geq 0$ and $\omega \in \mathbb{R}$, this means

$$
\left\langle\left(\left(\sigma^{2}-\omega^{2}\right) I+A_{0}\right) z, z\right\rangle-2 i \sigma \omega\|z\|^{2}+\frac{-\sigma+i \omega}{2}\left\|C_{0} z\right\|^{2}=0 .
$$


Looking at the imaginary part of this, we see that $\omega \neq 0$ implies

$$
-2 \sigma\|z\|^{2}+\frac{1}{2}\left\|C_{0} z\right\|^{2}=0,
$$

which is the same as the first equality in (4.7). Now we look at the real part of (4.8), using the expression for $\left\|C_{0} z\right\|^{2}$ that we have just found, obtaining (after a short computation) the second equality in (4.7).

Lemma 4.4. Suppose that $\omega \in \mathbb{R}$ is such that $i \omega \in \sigma_{p}(A)$. Then also $-i \omega \in \sigma_{p}(A)$ and $\omega^{2} \in \sigma_{p}\left(A_{0}\right)$. In this case, $z$ from (4.5) is an eigenvector of $A_{0}$ corresponding to the eigenvalue $\omega^{2}$ (in particular, $z \in H_{\alpha}$ for all $\alpha>0$ ) and we have $C_{0} z=0$.

Proof. Suppose that $i \omega \in \sigma_{p}(A)$, and let $z \in Z_{0}$ be the first component of a corresponding eigenvector as in (4.5). We know from (I.5.4) that $0 \in \rho(A)$ so that $\omega \neq 0$. According to the first part of (4.7) we have $C_{0} z=0$. Now (4.6) (with $\lambda=i \omega$ ) shows that $z$ is an eigenvector of $A_{0}$ corresponding to the eigenvalue $\omega^{2}$. It is now easy to see that the vector with components $z$ and $-i \omega z$ is also an eigenvector of $A$ corresponding to the eigenvalue $-i \omega$.

We denote by $\sigma_{a}(A)$ the set of those $\lambda \in \sigma(A)$ for which $\lambda$ is not an eigenvalue of $A$, but $\lambda I-A$ is not bounded from below. In other words, $\lambda \in \sigma_{a}(A)$ if $\lambda \notin \sigma_{p}(A)$ and there exists a sequence $\left(x_{n}\right)$ in $\mathcal{D}(A)$ with

$$
\left\|x_{n}\right\|_{X}=1 \quad \text { and } \quad \lim _{n \rightarrow \infty}\left\|(\lambda I-A) x_{n}\right\|_{X}=0 .
$$

Lemma 4.5. If $\omega \in \mathbb{R}$ is such that $i \omega \in \sigma(A)$, then

$$
i \omega \in \sigma_{p}(A) \cup \sigma_{a}(A) \text {. }
$$

Proof. Suppose that $i \omega \in \sigma(A)$. We prove that $i \omega \in \sigma_{p}(A) \cup \sigma_{a}(A)$ by showing that the contrary statement leads to a contradiction. Indeed, the contrary statement means that $i \omega I-A$ is bounded from below. In this case, the range of $i \omega I-A$ is not dense in $X$ (because if it were dense, then it were all of $X$, and hence $i \omega I-A$ would have a bounded inverse). Let $N$ be the orthogonal complement of the range of $i \omega I-A$; then it is easy to see that $N$ is invariant under $\mathbb{T}^{*}: \mathbb{T}_{t}^{*} N \subset N$ for all $t \geq 0$. Considering the restriction of $\mathbb{T}^{*}$ to $N$, we see that $\mathcal{D}\left(A^{*}\right) \cap N$ must be dense in $N$, so that, in particular, there exist elements $q \in \mathcal{D}\left(A^{*}\right) \cap N$ with $q \neq 0$. From the definition of $N$ we now see that for such $q$ we have $\left(-i \omega I-A^{*}\right) q=0$ so that $-i \omega \in \sigma_{p}\left(A^{*}\right)$. Introduce the isomorphism $J \in \mathcal{L}(X)$ defined by the matrix

$$
J=\left[\begin{array}{cc}
I & 0 \\
0 & -I
\end{array}\right]
$$

We have $J^{-1}=J$ and $A^{*}=J A J$; see the fourth step in the proof of Theorems I.1.1 and I.1.3 (in section I.6). Thus, $A$ and $A^{*}$ have the same eigenvalues so that $-i \omega \in \sigma_{p}(A)$. According to Lemma 4.4, we obtain that $i \omega \in \sigma_{p}(A)$, which contradicts our "contrary statement" at the beginning of this proof.

LEMmA 4.6. If $\omega \in \mathbb{R}$ is such that $i \omega \in \sigma_{a}(A)$, then also $-i \omega \in \sigma_{a}(A)$ and, moreover, $\omega^{2} \in \sigma_{p}\left(A_{0}\right) \cup \sigma_{a}\left(A_{0}\right)$.

Proof. Assume that $i \omega \in \sigma_{a}(A)$ so that for some sequence $\left(x_{n}\right)$ in $\mathcal{D}(A)$ we have (4.9) (with $\lambda=i \omega$ ). Denoting $x_{n}=\left[\begin{array}{l}z_{n} \\ w_{n}\end{array}\right]$ and $(i \omega I-A) x_{n}=\left[\begin{array}{l}\nu_{n} \\ \varepsilon_{n}\end{array}\right]$ so that $\nu_{n} \rightarrow 0$ (in $\left.H_{\frac{1}{2}}\right)$ and $\varepsilon_{n} \rightarrow 0$ (in $H$ ), we have

$$
\left[\begin{array}{cc}
i \omega I & -I \\
A_{0} & i \omega I+\frac{1}{2} B_{0} C_{0}
\end{array}\right]\left[\begin{array}{c}
z_{n} \\
w_{n}
\end{array}\right]=\left[\begin{array}{l}
\nu_{n} \\
\varepsilon_{n}
\end{array}\right]
$$


From the first row we have $w_{n}=i \omega z_{n}-\nu_{n}$. Substituting this into the equation representing the second row, we get

$$
A_{0} z_{n}-\omega^{2} z_{n}-i \omega \nu_{n}+\frac{i \omega}{2} B_{0} C_{0} z_{n}-\frac{1}{2} B_{0} C_{0} \nu_{n}=\varepsilon_{n}
$$

The two sides of this equation are in $H$, but some terms are in $H_{-\frac{1}{2}}$. Taking the scalar product with $z_{n}$ and using the duality pairing between $H_{-\frac{1}{2}}$ and $H_{\frac{1}{2}}$, we get

$$
\left\langle\left(A_{0}-\omega^{2} I\right) z_{n}, z_{n}\right\rangle-i \omega\left\langle\nu_{n}, z_{n}\right\rangle+\frac{i \omega}{2}\left\|C_{0} z_{n}\right\|^{2}-\frac{1}{2}\left\langle C_{0} \nu_{n}, C_{0} z_{n}\right\rangle=\left\langle\varepsilon_{n}, z_{n}\right\rangle,
$$

which shows that

$$
\lim _{n \rightarrow \infty}\left[\left\langle\left(A_{0}-\omega^{2} I\right) z_{n}, z_{n}\right\rangle+\frac{i \omega}{2}\left\|C_{0} z_{n}\right\|^{2}\right]=0 .
$$

Remember that $A$ is invertible (see (I.5.4)) so that $\omega \neq 0$. Taking the imaginary part of the last limit, we conclude that $\lim _{n \rightarrow \infty} C_{0} z_{n}=0$. Now going back to (4.10), we conclude that

$$
\lim _{n \rightarrow \infty}\left\|\left(\omega^{2} I-A_{0}\right) z_{n}\right\|_{-\frac{1}{2}}=0 .
$$

Now recall from (4.9) that

$$
\left\|x_{n}\right\|_{X}^{2}=\left\|z_{n}\right\|_{\frac{1}{2}}^{2}+\left\|w_{n}\right\|_{0}^{2}=1 .
$$

Since $w_{n}=i \omega z_{n}-\nu_{n}$ with $\lim _{n \rightarrow \infty}\left\|\nu_{n}\right\|_{\frac{1}{2}}=0$, we have $\lim _{n \rightarrow \infty}\left(\left\|w_{n}\right\|_{0}^{2}-\omega^{2}\left\|z_{n}\right\|_{0}^{2}\right)$ $=0$ so that $\lim _{n \rightarrow \infty}\left(\left\|z_{n}\right\|_{\frac{1}{2}}^{2}+\omega^{2}\left\|z_{n}\right\|_{0}^{2}\right)=1$. Since $\left\|z_{n}\right\|_{0} \leq\left\|A_{0}^{-\frac{1}{2}}\right\| \cdot\left\|z_{n}\right\|_{\frac{1}{2}}$ and $\left\|A_{0}^{-\frac{1}{2}}\right\|^{2}=\left\|A_{0}^{-1}\right\|$, we obtain $\liminf _{n \rightarrow \infty}\left(1+\omega^{2}\left\|A_{0}^{-1}\right\|\right) \cdot\left\|z_{n}\right\|_{\frac{1}{2}}^{2} \geq 1$ so that the sequence $\left(\left\|z_{n}\right\|_{\frac{1}{2}}\right)$ is eventually bounded from below:

$$
\left\|z_{n}\right\|_{\frac{1}{2}} \geq m>0 \quad \text { for } \quad n \geq n_{0}
$$

We have for $n \geq n_{0}$

$$
\begin{aligned}
m \leq\left\|z_{n}\right\|_{\frac{1}{2}} & =\left\|A_{0} z_{n}\right\|_{-\frac{1}{2}}=\left\|\left(\omega^{2} I-A_{0}\right) z_{n}-\omega^{2} z_{n}\right\|_{-\frac{1}{2}} \\
& \leq\left\|\left(\omega^{2} I-A_{0}\right) z_{n}\right\|_{-\frac{1}{2}}+\omega^{2}\left\|z_{n}\right\|_{-\frac{1}{2}} .
\end{aligned}
$$

Now (4.11) implies that the sequence $\left(\left\|z_{n}\right\|_{-\frac{1}{2}}\right)$ is eventually bounded from below. This together with (4.11) implies that $\omega^{2} I-A_{0}$ is not bounded from below as an (unbounded) operator on $H_{-\frac{1}{2}}$ (with domain $H_{\frac{1}{2}}$ ). Since $\omega^{2} I-A_{0}$ as an operator on $H_{-\frac{1}{2}}$ is isomorphic to $\omega^{2} I-A_{0}$ as an operator on $H$ (with domain $H_{1}$ ), we conclude that the latter is also not bounded from below. Thus, $\omega^{2} \in \sigma_{p}\left(A_{0}\right) \cup \sigma_{a}\left(A_{0}\right)$.

It remains to prove that $-i \omega \in \sigma_{a}(A)$. Define

$$
\xi_{n}=\left[\begin{array}{c}
z_{n} \\
-w_{n}
\end{array}\right] \in H_{\frac{1}{2}} \times H_{\frac{1}{2}}
$$


so that $\left\|\xi_{n}\right\|_{X}=\left\|x_{n}\right\|_{X}=1$. We will use the extension of $A$ which maps $X$ into $X_{-1}$. In particular, this extension maps $H_{\frac{1}{2}} \times H_{\frac{1}{2}} \subset X$ into $H_{\frac{1}{2}} \times H_{-\frac{1}{2}} \subset X_{-1}$, as it is easy to see (see Proposition I.5.2 for the inclusion $H_{\frac{1}{2}} \times H_{-\frac{1}{2}} \subset X_{-1}$ ). We have

$$
(-i \omega I-A) \xi_{n}=\left[\begin{array}{cc}
-i \omega I & -I \\
A_{0} & -i \omega I+\frac{1}{2} B_{0} C_{0}
\end{array}\right]\left[\begin{array}{c}
z_{n} \\
-w_{n}
\end{array}\right]=\left[\begin{array}{c}
-\nu_{n} \\
\varphi_{n}
\end{array}\right]
$$

where $\varphi_{n} \in H_{-\frac{1}{2}}$ is given by a formula similar to (4.10):

$$
\varphi_{n}=\left(A_{0}-\omega^{2} I\right) z_{n}-i \omega \nu_{n}-\frac{i \omega}{2} B_{0} C_{0} z_{n}+\frac{1}{2} B_{0} C_{0} \nu_{n} .
$$

Remember that $\nu_{n} \rightarrow 0$ (in $H_{\frac{1}{2}}$ ) and $C_{0} z_{n} \rightarrow 0$ (in $U$ ). Using also (4.11), we conclude from the above formula that $\varphi_{n} \rightarrow 0$ (in $H_{-\frac{1}{2}}$ ). From here we see that

$$
\lim _{n \rightarrow \infty}(-i \omega I-A) \xi_{n}=0 \quad \text { in } H_{\frac{1}{2}} \times H_{-\frac{1}{2}} .
$$

Since the sequence $\left(\xi_{n}\right)$ is bounded from below in $H_{\frac{1}{2}} \times H_{\frac{1}{2}}$, it follows that $(-i \omega I-A)$ is not bounded from below (as an operator from $H_{\frac{1}{2}} \times H_{\frac{1}{2}}$ to $H_{\frac{1}{2}} \times H_{-\frac{1}{2}}$ ). According to point (1) of Proposition I.5.3, it follows that $-i \omega \in \sigma(A)$. Now we can apply Lemma 4.5 to conclude that $-i \omega \in \sigma_{p}(A) \cup \sigma_{a}(A)$. If we would have $-i \omega \in \sigma_{p}(A)$, then it would follow from Lemma 4.4 that also $i \omega \in \sigma_{p}(A)$, which would contradict our assumption that $i \omega \in \sigma_{a}(A)$. Thus, we must have $-i \omega \in \sigma_{a}(A)$.

Proof of Theorem 1.5. According to Lemmas 4.4, 4.5, and 4.6, if $\omega \in \mathbb{R}$ is such that $i \omega \in \sigma(A)$, then $\omega^{2} \in \sigma\left(A_{0}\right)$. Thus, if $\sigma\left(A_{0}\right)$ is countable, as assumed in the theorem, then also $\sigma(A) \cap i \mathbb{R}$ must be countable, as required in Proposition 3.4. Now the equivalence of points (1)-(7) in the theorem follows from Proposition 3.4.

It remains to prove the equivalence between point (8) and the other points. Suppose that (8) holds. We claim that $A$ has no eigenvalues on the imaginary axis. Indeed, if $\omega \in \mathbb{R}$ were such that $i \omega \in \sigma_{p}(A)$, then according to Lemma $4.4 \omega^{2} \in \sigma_{p}\left(A_{0}\right)$ and for a corresponding eigenvector $z \in H_{1}$ we would have $C_{0} z=0$, which would contradict (8). Now we can apply the main theorem of Arendt and Batty [2]: our earlier claim together with the fact that $\sigma(A) \cap i \mathbb{R}$ is countable implies that $\mathbb{T}$ is strongly stable. Thus, point (1) of the theorem holds and with it all the others.

Conversely, suppose that point (8) is false, i.e., there exists an $\omega \in \mathbb{R}$ such that $\omega^{2} \in \sigma_{p}\left(A_{0}\right)$, and for a corresponding eigenvector $z \in H_{1}$ we have $C_{0} z=0$. Let $x$ be defined by (4.5) with $\lambda=i \omega$. Then it is easy to verify that $x \in \mathcal{D}(A)$ and $A x=i \omega x$, whence $\mathbb{T}_{t} x=e^{i \omega t} x$. This shows that $\mathbb{T}$ is not strongly stable, so in this case the points (1)-(7) are all false.

5. Examples. The aim of this section is to apply the general stability results derived earlier to some models based on PDEs. We will consider an Euler-Bernoulli beam with an exponentially stabilizing feedback acting in one point followed by an $n$-dimensional wave equation with boundary control and a nonlocal feedback term entering a Dirichlet boundary condition.

5.1. An Euler-Bernoulli beam with pointwise control. The physical system that we have in mind consists of two rigidly joined beams with both velocity and angular velocity damping at the joint. The other end of both beams is hinged. The inputs are a force and a torque acting at the joint (in addition to the damping force and torque), and the measurements depend linearly on the velocity and the angular 
velocity at the joint as well as on the inputs. The fact that we apply both a force and a torque feedback at the joint prevents the well-known lack of robustness of stability concerning the location of the joint: we obtain exponential stability for any location. For the case when only force feedback or only torque feedback is applied, lack of robustness of exponential stability was demonstrated in Rebarber [26]. (We refer to [26] for earlier references on this subject.)

Our system consists of two homogeneous Euler-Bernoulli beams situated along the intervals $[0, \xi]$ and $[\xi, \pi]$ with the joint at the point $\xi \in(0, \pi)$. Denoting by $[f]_{\xi}$ the jump of the function $f$ at $x=\xi$, we get the equations

$$
\begin{gathered}
\frac{\partial^{2} z}{\partial t^{2}}+\frac{\partial^{4} z}{\partial x^{4}}=0, \quad x \in(0, \pi) \backslash\{\xi\}, \quad[z]_{\xi}=0, \quad\left[\frac{\partial z}{\partial x}\right]_{\xi}=0 \\
{\left[\frac{\partial^{3} z}{\partial x^{3}}\right]_{\xi}+\frac{\alpha^{2}}{2} \frac{\partial z}{\partial t}(\xi, t)=\alpha u_{1}(t), \quad-\left[\frac{\partial^{2} z}{\partial x^{2}}\right]_{\xi}+\frac{\beta^{2}}{2} \frac{\partial^{2} z}{\partial x \partial t}(\xi, t)=\beta u_{2}(t),} \\
{\left[\frac{\partial^{3} z}{\partial x^{3}}\right]_{\xi}-\frac{\alpha^{2}}{2} \frac{\partial z}{\partial t}(\xi, t)=\alpha y_{1}(t), \quad-\left[\frac{\partial^{2} z}{\partial x^{2}}\right]_{\xi}-\frac{\beta^{2}}{2} \frac{\partial^{2} z}{\partial x \partial t}(\xi, t)=\beta y_{2}(t),} \\
z(x, 0)=z_{0}(x), \quad \frac{\partial z}{\partial t}(x, 0)=w_{0}(x), \quad x \in(0, \pi) \backslash\{\xi\} \\
z(0, t)=z(\pi, t)=0, \quad \frac{\partial^{2} z}{\partial x^{2}}(0, t)=\frac{\partial^{2} z}{\partial x^{2}}(\pi, t)=0
\end{gathered}
$$

Here, $z$ stands for the transverse displacement of the beam and $\alpha, \beta>0$ are damping coefficients. The external force is $\alpha u_{1}$ and the external torque is $\beta u_{2}$, both acting at $\xi$. The output signals are $y_{1}$ and $y_{2}$.

An equivalent formulation of (5.1)-(5.5) can be obtained by considering a single homogeneous Euler-Bernoulli beam situated along the interval $[0, \pi]$ with a force and a torque acting at the point $\xi \in(0, \pi)$. (The equivalence is proved in Proposition 5.2 below.) In this case the equations (5.1)-(5.2) are replaced by

$$
\begin{gathered}
\frac{\partial^{2} z}{\partial t^{2}}+\frac{\partial^{4} z}{\partial x^{4}}+\frac{\alpha^{2}}{2} \frac{\partial}{\partial t}(z(\xi, t)) \delta_{\xi}-\frac{\beta^{2}}{2} \frac{\partial}{\partial t}\left(\frac{\partial z}{\partial x}(\xi, t)\right) \frac{\mathrm{d} \delta_{\xi}}{\mathrm{d} x} \\
=\alpha u_{1}(t) \delta_{\xi}-\beta u_{2}(t) \frac{\mathrm{d} \delta_{\xi}}{\mathrm{d} x} .
\end{gathered}
$$

Here, $\delta_{\xi}$ is the Dirac mass concentrated at $\xi$ and (5.6) is understood as an equation in $\mathcal{D}^{\prime}(0, \pi)$ (distributions on $(0, \pi)$ ). The outputs are equivalently given by

$$
y_{1}(t)=-\alpha \frac{\partial z}{\partial t}(\xi, t)+u_{1}(t), \quad y_{2}(t)=-\beta \frac{\partial^{2} z}{\partial t \partial x}(\xi, t)+u_{2}(t) .
$$

The well-posedness of the system described by (5.4)-(5.7) can be obtained by using Theorem 1.1 if we introduce the appropriate spaces and operators. We start by defining $H=L^{2}[0, \pi]$ and the operator $A_{0}: \mathcal{D}\left(A_{0}\right) \rightarrow H$ by

$$
\mathcal{D}\left(A_{0}\right)=\left\{\phi \in \mathcal{H}^{4}(0, \pi) \mid \phi(0)=\phi(\pi)=0, \frac{\mathrm{d}^{2} \phi}{\mathrm{d} x^{2}}(0)=\frac{\mathrm{d}^{2} \phi}{\mathrm{d} x^{2}}(\pi)=0\right\},
$$




$$
A_{0} \phi=\frac{\mathrm{d}^{4} \phi}{\mathrm{d} x^{4}} \quad \forall \phi \in \mathcal{D}\left(A_{0}\right) .
$$

It is well known that $A_{0}$ is self-adjoint, positive, and boundedly invertible. As in section 1 , we put $H_{1}=\mathcal{D}\left(A_{0}\right)$ and we introduce the spaces $H_{\alpha}(\alpha \in \mathbb{R})$ by considering fractional powers of $A_{0}$ and duality. A simple calculation shows that

$$
H_{\frac{1}{2}}=\mathcal{D}\left(A_{0}^{\frac{1}{2}}\right)=\mathcal{H}^{2}(0, \pi) \cap \mathcal{H}_{0}^{1}(0, \pi)
$$

with the norm

$$
\|z\|_{\frac{1}{2}}^{2}=\int_{0}^{\pi}\left|\frac{\mathrm{d}^{2} z}{\mathrm{~d} x^{2}}(x)\right|^{2} \mathrm{~d} x
$$

Proposition 5.1. The equations (5.4)-(5.7) determine a conservative linear system $\Sigma$ with input and output space $\mathbb{C}^{2}$ and with state space $X=H_{\frac{1}{2}} \times H$. For $z_{0} \in H_{\frac{1}{2}}, w_{0} \in H$, and $u_{1}, u_{2} \in L^{2}[0, \infty)$ the equations (5.4)-(5.6) admit a unique solution

$$
z \in B C\left(0, \infty ; H_{\frac{1}{2}}\right) \cap B C^{1}(0, \infty ; H) \cap \mathcal{H}_{l o c}^{2}\left(0, \infty ; H_{-\frac{1}{2}}\right) .
$$

Moreover, we have $z(\xi, \cdot), \frac{\partial z}{\partial x}(\xi, \cdot) \in \mathcal{H}^{1}(0, \infty)$.

Proof. We take $U=\mathbb{C}^{2}$, and $B_{0} \in \mathcal{L}\left(U, H_{-\frac{1}{2}}\right)$ is defined by

$$
B_{0}\left[\begin{array}{l}
\mathrm{v}_{1} \\
\mathrm{v}_{2}
\end{array}\right]=\alpha \mathrm{v}_{1} \delta_{\xi}-\beta \mathrm{v}_{2} \frac{\mathrm{d} \delta_{\xi}}{\mathrm{d} x} \quad \forall\left[\begin{array}{l}
\mathrm{v}_{1} \\
\mathrm{v}_{2}
\end{array}\right] \in \mathbb{C}^{2}
$$

By the definition of $B_{0}$, for each $\mathrm{v}_{1}, \mathrm{v}_{2} \in \mathbb{C}, B_{0}\left[\begin{array}{l}\mathrm{v}_{1} \\ \mathrm{v}_{2}\end{array}\right]$ is a bounded linear functional acting on $H_{\frac{1}{2}}$ so that indeed $B_{0}$ maps $\mathbb{C}^{2}$ into $H_{-\frac{1}{2}}$. The adjoint of $B_{0}$ is

$$
C_{0} \phi=B_{0}^{*} \phi=\left[\begin{array}{c}
\alpha \phi(\xi) \\
\beta \frac{\mathrm{d} \phi}{\mathrm{d} x}(\xi)
\end{array}\right] \quad \forall \phi \in H_{\frac{1}{2}} .
$$

Now it is clear that the problem (5.4)-(5.7) can be written in the form (1.1)-(1.3). Thus, this proposition follows from Theorem 1.1.

Note that since $\Sigma$ is conservative, for every $t \geq 0$ we have

$$
\begin{aligned}
\left\|\left[\begin{array}{c}
z(t) \\
\dot{z}(t)
\end{array}\right]\right\|_{X}^{2}-\left\|\left[\begin{array}{c}
z(0) \\
\dot{z}(0)
\end{array}\right]\right\|_{X}^{2} & =\int_{0}^{t}\left[\left|u_{1}(\sigma)\right|^{2}+\left|u_{2}(\sigma)\right|^{2}\right] \mathrm{d} \sigma \\
& -\int_{0}^{t}\left[\left|y_{1}(\sigma)\right|^{2}+\left|y_{2}(\sigma)\right|^{2}\right] \mathrm{d} \sigma .
\end{aligned}
$$

The space $Z_{0}$ defined after (1.5) (see also Theorem I.1.2) is now given by

$$
Z_{0}=\left\{z \in H_{\frac{1}{2}}|z|_{(0, \xi)} \in \mathcal{H}^{4}(0, \xi),\left.z\right|_{(\xi, 1)} \in \mathcal{H}^{4}(\xi, 1)\right\} .
$$

The systems (5.1)-(5.5) and (5.4)-(5.7) are equivalent in the following sense.

Proposition 5.2. Consider the functions

$$
z \in C\left(0, \infty ; Z_{0}\right) \cap C^{1}\left(0, \infty ; H_{\frac{1}{2}}\right) \cap C^{2}(0, \infty ; H), \quad u, y \in C(0, \infty ; U) .
$$

Then $z, u$, and $y$ satisfy (5.1)-(5.5) if and only if they satisfy (5.4)-(5.7). 
Proof. We denote by $z^{(\mathrm{iii})}$ (respectively, by $z^{(\mathrm{iv})}$ ) the third (respectively, the fourth) derivative of $z$ computed in $\mathcal{D}^{\prime}((0, \pi) \backslash\{\xi\})$. Roughly speaking, these derivatives are calculated almost everywhere, ignoring the possible jumps at $x=\xi$. Hence they are, in general, different from $\frac{\mathrm{d}^{3} z}{\mathrm{~d} x^{3}}$ and $\frac{\mathrm{d}^{4} z}{\mathrm{~d} x^{4}}$ computed in the sense of distributions, i.e., in $\mathcal{D}^{\prime}(0, \pi)$. We define $L_{0} \in \mathcal{L}\left(Z_{0}, L^{2}[0, \pi]\right)$ and $G_{0} \in \mathcal{L}\left(Z_{0}, U\right)$ by

$$
L_{0} z=z^{(\mathrm{iv})} \text { and } G_{0} z=\left[\frac{1}{\alpha}\left[z^{\mathrm{iii}}\right]_{\xi}-\frac{1}{\beta}\left[\frac{d^{2} z}{d x^{2}}\right]_{\xi}\right]^{T}
$$

( $T$ stands for transpose). Simple calculations show that

$$
L_{0}=A_{0}-B_{0} G_{0}, \quad G_{0} H_{1}=\{0\}, \quad G_{0} A_{0}^{-1} B_{0}=I .
$$

By Theorem I.1.4 we obtain that the functions $z, u, y$ satisfy (5.4)-(5.7) (which are the same, in this example, as (1.1)-(1.3)) if and only if they satisfy

$$
\ddot{z}(t)+L_{0} z(t)=0, \quad G_{0} z(t)+\frac{1}{2} C_{0} \dot{z}(t)=u(t), \quad G_{0} z(t)-\frac{1}{2} C_{0} \dot{z}(t)=y(t)
$$

(which are the same as (5.1)-(5.3) and (5.5)) as well as (5.4).

The main result of this section is the following.

THEOREM 5.3. For all $\xi \in(0, \pi),(5.4)-(5.7)$ define an exponentially stable wellposed system with input and output space $U=\mathbb{C}^{2}$ and state space $X$.

Proof. Let $A: \mathcal{D}(A) \rightarrow X$ be defined as in (1.7), (1.8). If $\psi=\left[\begin{array}{c}z \\ w\end{array}\right] \in \mathcal{D}(A)$, then $w \in H_{\frac{1}{2}}$ and $z$ is piecewise in $H^{4}$ (on $(0, \xi)$ and on $(\xi, \pi)$ ). Thus, $\mathcal{D}(A)$ is compactly included in $X$ so that $A$ has a compact resolvent, hence $\sigma(A)$ consists of eigenvalues of $A$. We prove that $\sigma(A) \cap i \mathbb{R}=\emptyset$. Arguing by contradiction, suppose that $i \beta \in \sigma(A)$ with $\beta \in \mathbb{R}$. According to Lemma 4.4, if $\psi$ is an eigenvector of $A$ corresponding to $i \beta$, then $\psi=\left[\begin{array}{c}z \\ i \beta z\end{array}\right]$, where $\left(\beta^{2} I-A_{0}\right) z=0$. This implies that

$$
\frac{\mathrm{d}^{4} z}{\mathrm{~d} x^{4}}-\beta^{2} z=0, \quad z(0)=z(\pi)=0, \quad \frac{\mathrm{d}^{2} z}{\mathrm{~d} x^{2}}(0)=\frac{\mathrm{d}^{2} z}{\mathrm{~d} x^{2}}(\pi)=0 .
$$

It follows that $z(x)=c \sin (n x)$ for some $c \in \mathbb{C}$ and $n \in \mathbb{N}$ (with $n^{4}=\beta^{2}$ ). Moreover, by Lemma 4.4 we also have $C_{0} z=0$ which, by (5.8), yields that $z(\xi)=0, \frac{\mathrm{d} z}{\mathrm{~d} x}(\xi)=0$. This implies that $z=0$, which is a contradiction. Thus, $\sigma(A) \cap i \mathbb{R}=\emptyset$.

By the equivalence of (3) and (9) in Theorem 1.3, it suffices to show that

$$
\sup _{\omega \in \mathbb{R}}\|\omega V(i \omega)\|_{\mathcal{L}(H)}<\infty .
$$

Suppose that this condition is false. By the uniform boundedness theorem, there exist a sequence of real numbers $\left(\beta_{n}\right)$ with $\left|\beta_{n}\right| \rightarrow \infty$ and $h \in H$ such that $\lambda_{n}=$ $\left\|\beta_{n} V\left(i \beta_{n}\right) h\right\| \rightarrow \infty$. Denoting $z_{n}=\frac{1}{\lambda_{n}} V\left(i \beta_{n}\right) h$ and $g_{n}=\frac{1}{\lambda_{n}} h$ (so that $g_{n} \rightarrow 0$ in $H$ ), we have

$$
\begin{gathered}
\left\|\beta_{n} z_{n}\right\|_{H}=1 \quad \forall n \in \mathbb{N}, \\
-\beta_{n}^{2} z_{n}+A_{0} z_{n}+\frac{i \beta_{n}}{2} B_{0} C_{0} z_{n}=g_{n} \in H .
\end{gathered}
$$

We show (in four steps) that (5.12) and (5.13) lead to a contradiction. 
First step. Taking the inner product in $H$ of the sides of (5.13) with $\beta_{n} z_{n}$, taking the imaginary parts, and using (5.12), we obtain that $\beta_{n} C_{0} z_{n} \rightarrow 0$. According to (5.8) this means

$$
\left|\beta_{n} z_{n}(\xi)\right| \rightarrow 0, \quad\left|\beta_{n} \frac{\mathrm{d} z_{n}}{\mathrm{~d} x}(\xi)\right| \rightarrow 0 .
$$

On the other hand, (5.13) implies that $z_{n} \in Z_{0}$ so that by (5.10) we have

$$
A_{0} z_{n}=L_{0} z_{n}+B_{0} G_{0} z_{n} .
$$

Substituting this into (5.13) and using the fact that $B_{0} u \in H$ iff $u=0$, we obtain

$$
-\beta_{n}^{2} z_{n}+L_{0} z_{n}=g_{n} \text { and } G_{0} z_{n}=\frac{-i \beta_{n}}{2} C_{0} z_{n} .
$$

The above relations together with (5.8) and (5.9) imply that

$$
\begin{gathered}
-\beta_{n}^{2} z_{n}+z_{n}^{(\mathrm{iv})}=g_{n} \rightarrow 0 \text { in } L^{2}[0, \pi], \\
z_{n}^{(\mathrm{iii})}\left(\xi^{+}\right)-z_{n}^{(\mathrm{iii})}\left(\xi^{-}\right)=-i \beta_{n} \frac{\alpha^{2}}{2} z_{n}(\xi), \\
\frac{\mathrm{d}^{2} z_{n}}{\mathrm{~d} x^{2}}\left(\xi^{+}\right)-\frac{\mathrm{d}^{2} z_{n}}{\mathrm{~d} x^{2}}\left(\xi^{-}\right)=i \beta_{n} \frac{\beta^{2}}{2} \frac{\mathrm{d} z_{n}}{\mathrm{~d} x}(\xi) .
\end{gathered}
$$

Second step. Define $f_{n} \in H$ by $f_{n}(x)=x \frac{\mathrm{d} z_{n}}{\mathrm{~d} x}(x)$ for $x \in[0, \xi]$ and $f_{n}(x)=0$ for $x>\xi$. We take the inner product of the sides of (5.15) with $f_{n}$ to get

$$
\int_{0}^{\xi}\left(-\beta_{n}^{2} z_{n}(x)+z_{n}^{(\mathrm{iv})}(x)\right) x \frac{\mathrm{d} \bar{z}_{n}}{\mathrm{~d} x}(x) \mathrm{d} x=\int_{0}^{\xi} x g_{n}(x) \frac{\mathrm{d} \bar{z}_{n}}{\mathrm{~d} x}(x) \mathrm{d} x .
$$

By a straightforward calculation, the terms on the left-hand side become

$$
\begin{aligned}
\operatorname{Re}\left\{\int_{0}^{\xi}-\beta_{n}^{2} z_{n}(x) x \frac{\mathrm{d} \bar{z}_{n}}{\mathrm{~d} x}(x) \mathrm{d} x\right\} & =-\frac{1}{2} \xi\left|\beta_{n} z_{n}(\xi)\right|^{2}+\frac{1}{2} \int_{0}^{\xi}\left|\beta_{n} z_{n}(x)\right|^{2} \mathrm{~d} x, \\
\operatorname{Re}\left\{\int_{0}^{\xi} z_{n}^{(\mathrm{iv})}(x) x \frac{\mathrm{d} \bar{z}_{n}}{\mathrm{~d} x}(x) \mathrm{d} x\right\} & =\operatorname{Re}\left[\left(\xi z_{n}^{(\mathrm{iii})}\left(\xi^{-}\right)-\frac{\mathrm{d}^{2} z_{n}}{\mathrm{~d} x^{2}}\left(\xi^{-}\right)\right) \frac{\mathrm{d} \bar{z}_{n}}{\mathrm{~d} x}(\xi)\right] \\
& -\frac{\xi}{2}\left|\frac{\mathrm{d}^{2} z_{n}}{\mathrm{~d} x^{2}}\left(\xi^{-}\right)\right|^{2}+\frac{3}{2} \int_{0}^{\xi}\left|\frac{\mathrm{d}^{2} z_{n}}{\mathrm{~d} x^{2}}(x)\right|^{2} \mathrm{~d} x .
\end{aligned}
$$

The two relations above and (5.18) yield

$$
\begin{gathered}
-\frac{1}{2} \xi\left|\beta_{n} z_{n}(\xi)\right|^{2}+\frac{1}{2} \int_{0}^{\xi}\left|\beta_{n} z_{n}(x)\right|^{2} \mathrm{~d} x+\operatorname{Re}\left[\left(\xi z_{n}^{(\mathrm{iii})}\left(\xi^{-}\right)-\frac{\mathrm{d}^{2} z_{n}}{\mathrm{~d} x^{2}}\left(\xi^{-}\right)\right) \frac{\mathrm{d} \bar{z}_{n}}{\mathrm{~d} x}(\xi)\right] \\
-\frac{\xi}{2}\left|\frac{\mathrm{d}^{2} z_{n}}{\mathrm{~d} x^{2}}\left(\xi^{-}\right)\right|^{2}+\frac{3}{2} \int_{0}^{\xi}\left|\frac{\mathrm{d}^{2} z_{n}}{\mathrm{~d} x^{2}}(x)\right|^{2} \mathrm{~d} x=\operatorname{Re} \int_{0}^{\xi} x g_{n}(x) \frac{\mathrm{d} \bar{z}_{n}}{\mathrm{~d} x}(x) \mathrm{d} x .
\end{gathered}
$$


Since $g_{n}$ converges to zero in $L^{2}[0, \xi]$, we deduce that the right-hand side of the last formula converges to zero. This implies that

$$
\begin{aligned}
-\frac{1}{2} \xi\left|\beta_{n} z_{n}(\xi)\right|^{2}+ & \frac{1}{2} \int_{0}^{\xi}\left|\beta_{n} z_{n}(x)\right|^{2} \mathrm{~d} x+\operatorname{Re}\left[\left(\xi z_{n}^{(\mathrm{iii})}\left(\xi^{-}\right)-\frac{\mathrm{d}^{2} z_{n}}{\mathrm{~d} x^{2}}\left(\xi^{-}\right)\right) \frac{\mathrm{d} \bar{z}_{n}}{\mathrm{~d} x}(\xi)\right] \\
& -\frac{\xi}{2}\left|\frac{\mathrm{d}^{2} z_{n}}{\mathrm{~d} x^{2}}\left(\xi^{-}\right)\right|^{2}+\frac{3}{2} \int_{0}^{\xi}\left|\frac{\mathrm{d}^{2} z_{n}}{\mathrm{~d} x^{2}}(x)\right|^{2} \mathrm{~d} x \rightarrow 0 .
\end{aligned}
$$

On the other hand, from (5.15) and the fact that $\left\|\beta_{n} z_{n}\right\|_{H}=1$ we deduce that $\left(z_{n}^{(\mathrm{iv})} / \beta_{n}\right)$ is a bounded sequence in $L^{2}[0, \xi]$. This further implies the boundedness of the sequences of complex numbers $\left(z_{n}^{(\mathrm{iii})}\left(\xi^{-}\right) / \beta_{n}\right)$ and $\left(\frac{\mathrm{d}^{2} z_{n}}{\mathrm{~d} x^{2}}\left(\xi^{-}\right) / \beta_{n}\right)$. Then, by $(5.14)$ we obtain that

$$
-\frac{1}{2} \xi\left|\beta_{n} z_{n}(\xi)\right|^{2}+\operatorname{Re}\left[\left(\xi z_{n}^{(\mathrm{iii})}\left(\xi^{-}\right)-\frac{\mathrm{d}^{2} z_{n}}{\mathrm{~d} x^{2}}\left(\xi^{-}\right)\right) \frac{\mathrm{d} \bar{z}_{n}}{\mathrm{~d} x}(\xi)\right] \rightarrow 0 .
$$

Relations (5.19) and (5.20) lead to

$$
\frac{1}{2} \int_{0}^{\xi}\left|\beta_{n} z_{n}(x)\right|^{2} \mathrm{~d} x+\frac{3}{2} \int_{0}^{\xi}\left|\frac{\mathrm{d}^{2} z_{n}}{\mathrm{~d} x^{2}}\right|^{2} \mathrm{~d} x-\frac{\xi}{2}\left|\frac{\mathrm{d}^{2} z_{n}}{\mathrm{~d} x^{2}}\left(\xi^{-}\right)\right|^{2} \rightarrow 0 .
$$

Similarly, we take the inner product of the sides of (5.15) with $(x-\pi) \frac{\mathrm{d} z_{n}}{\mathrm{~d} x}$ truncated to $[\xi, \pi]$, and then we repeat the above argument. This gives

$$
\frac{1}{2} \int_{\xi}^{\pi}\left|\beta_{n} z_{n}(x)\right|^{2} \mathrm{~d} x+\frac{3}{2} \int_{\xi}^{\pi}\left|\frac{\mathrm{d}^{2} z_{n}}{\mathrm{~d} x^{2}}\right|^{2} \mathrm{~d} x-\frac{\pi-\xi}{2}\left|\frac{\mathrm{d}^{2} z_{n}}{\mathrm{~d} x^{2}}\left(\xi^{+}\right)\right|^{2} \rightarrow 0 .
$$

If we add the last two formulas and we use (5.13), we obtain

$$
\begin{aligned}
& \int_{0}^{\pi}\left|\beta_{n} z_{n}(x)\right|^{2} \mathrm{~d} x+3 \int_{0}^{\pi}\left|\frac{\mathrm{d}^{2} z_{n}}{\mathrm{~d} x^{2}}(x)\right|^{2} \mathrm{~d} x \\
& -\frac{\xi}{2}\left|\frac{\mathrm{d}^{2} z_{n}}{\mathrm{~d} x^{2}}\left(\xi^{-}\right)\right|^{2}-\frac{\pi-\xi}{2}\left|\frac{\mathrm{d}^{2} z_{n}}{\mathrm{~d} x^{2}}\left(\xi^{+}\right)\right|^{2} \rightarrow 0 .
\end{aligned}
$$

Third step. We show that both $\left|\frac{\mathrm{d}^{2} z_{n}}{\mathrm{~d} x^{2}}\left(\xi^{-}\right)\right|$and $\left|\frac{\mathrm{d}^{2} z_{n}}{\mathrm{~d} x^{2}}\left(\xi^{+}\right)\right|$converge to zero. We use an idea of Liu; see, for instance, [1]: we take the inner product of the sides of (5.15) with $\frac{1}{\phi_{n}} e^{-\phi_{n}(\xi-x)}$ truncated to $[0, \xi]$, where $\phi_{n}=\sqrt{\left|\beta_{n}\right|}$. Thus, we obtain

$$
-\int_{0}^{\xi} \phi_{n}^{3} z_{n}(x) e^{-\phi_{n}(\xi-x)} \mathrm{d} x+\int_{0}^{\xi} \frac{z_{n}^{(\mathrm{iv})}(x)}{\phi_{n}} e^{-\phi_{n}(\xi-x)} \mathrm{d} x=\int_{0}^{\xi} g_{n}(x) \frac{e^{-\phi_{n}(\xi-x)}}{\phi_{n}} \mathrm{~d} x .
$$

By using (5.12), (5.15) again and the fact that $\phi_{n} e^{-\phi_{n}(\xi-x)}$ is bounded in $L^{1}[0, \pi]$, we obtain that

$$
-\int_{0}^{\xi} \phi_{n}^{3} z_{n}(x) e^{-\phi_{n}(\xi-x)} \mathrm{d} x+\int_{0}^{\xi} \frac{z_{n}^{(\mathrm{iv})}(x)}{\phi_{n}} e^{-\phi_{n}(\xi-x)} \mathrm{d} x \rightarrow 0 .
$$

Integrating by parts the second term above four times, we obtain

$$
\int_{0}^{\xi} \frac{z_{n}^{(\mathrm{iv})}(x)}{\phi_{n}} e^{-\phi_{n}(\xi-x)} \mathrm{d} x
$$




$$
=\left.\left(\frac{z_{n}^{(\mathrm{iii})}(x)}{\phi_{n}}-\frac{\mathrm{d}^{2} z_{n}}{\mathrm{~d} x^{2}}(x)+\phi_{n} \frac{\mathrm{d} z_{n}}{\mathrm{~d} x}(x)-\phi_{n}^{2} z_{n}\right) e^{-\phi_{n}(\xi-x)}\right|_{0} ^{\xi}+\int_{0}^{\xi} \phi_{n}^{3} z_{n} e^{-\phi_{n}(\xi-x)} \mathrm{d} x .
$$

By using (5.22) we obtain from here that

$$
\left.\left(\frac{z_{n}^{(\mathrm{iii})}(x)}{\phi_{n}}-\frac{\mathrm{d}^{2} z_{n}}{\mathrm{~d} x^{2}}(x)+\phi_{n} \frac{\mathrm{d} z_{n}}{\mathrm{~d} x}(x)-\phi_{n}^{2} z_{n}\right) e^{-\phi_{n}(\xi-x)}\right|_{0} ^{\xi} \rightarrow 0 .
$$

Among the boundary terms in (5.23), those at $x=0$ all converge to zero due to the exponential decay of $e^{-\phi_{n} \xi}$ and to the boundedness of $\left(z_{n}^{\text {(iv) }} / \beta_{n}\right)$ in $L^{2}[0, \xi]$; the terms containing $z_{n}(\xi), \frac{\mathrm{d} z_{n}}{\mathrm{~d} x}(\xi)$ also converge to zero due to (5.14). Thus, (5.23) yields

$$
\frac{z_{n}^{(\mathrm{iii})}}{\phi_{n}}\left(\xi^{-}\right)-\frac{\mathrm{d}^{2} z_{n}}{\mathrm{~d} x^{2}}\left(\xi^{-}\right) \rightarrow 0 .
$$

Similarly, we take the inner product of the sides of (5.15) with $\frac{1}{\phi_{n}} e^{-\phi_{n}(x-\xi)}$ in $L^{2}[\xi, \pi]$. Repeating the above analysis, we obtain

$$
\frac{z_{n}^{(\mathrm{iii})}}{\phi_{n}}\left(\xi^{+}\right)+\frac{\mathrm{d}^{2} z_{n}}{\mathrm{~d} x^{2}}\left(\xi^{+}\right) \rightarrow 0
$$

On the other hand, by using (5.14) and (5.16), we obtain

$$
z_{n}^{(\mathrm{iii})}\left(\xi^{+}\right)-z_{n}^{(\mathrm{iii})}\left(\xi^{-}\right) \rightarrow 0 .
$$

Then the difference of (5.24) and (5.25) leads, by using (5.26), to

$$
\frac{\mathrm{d}^{2} z_{n}}{\mathrm{~d} x^{2}}\left(\xi^{+}\right)+\frac{\mathrm{d}^{2} z_{n}}{\mathrm{~d} x^{2}}\left(\xi^{-}\right) \rightarrow 0
$$

Recall that, by (5.14) and (5.17), we have $\frac{\mathrm{d}^{2} z_{n}}{\mathrm{~d} x^{2}}\left(\xi^{+}\right)-\frac{\mathrm{d}^{2} z_{n}}{\mathrm{~d} x^{2}}\left(\xi^{-}\right) \rightarrow 0$. Therefore,

$$
\frac{\mathrm{d}^{2} z_{n}}{\mathrm{~d} x^{2}}\left(\xi^{-}\right) \rightarrow 0, \quad \frac{\mathrm{d}^{2} z_{n}}{\mathrm{~d} x^{2}}\left(\xi^{+}\right) \rightarrow 0 .
$$

Fourth step. Relations (5.21) and (5.27) imply that $\left\|\beta_{n} z_{n}\right\|_{H} \rightarrow 0$, which clearly contradicts (5.12). This contradiction shows that (5.11) holds.

Theorem 5.3 can be generalized for coupled beams described by a version of (5.6) containing variable coefficients; see Ammari, Liu, and Tucsnak [1].

Theorem 5.3 together with Theorem 1.3 implies the following.

Proposition 5.4. The system from Theorem 5.3 is exactly controllable.

5.2. The wave equation with Dirichlet-type nonlocal boundary feedback. The physical system that we have in mind consists of a vibrating membrane which is fixed on a part of the boundary, while on the other part of the boundary the vibrations are damped by a feedback control acting on the Dirichlet boundary condition. The input is the displacement field on the controlled part of the boundary, and the measurement depends linearly on the velocity field as well as on the input. A membrane could be modelled in a domain in $\mathbb{R}^{2}$, but we consider a more general wave equation on an $n$-dimensional (possibly unbounded) domain $\Omega$. The boundary $\Gamma$ of $\Omega \subset \mathbb{R}^{n}$ is assumed to be of class $C^{2}$ and to have a decomposition 
as $\Gamma=\overline{\Gamma_{0} \cup \Gamma_{1}}$, where $\Gamma_{0}, \Gamma_{1}$ are disjoint open parts of $\Gamma$ with $\Gamma_{1} \neq \emptyset$. We also assume that the Poincaré inequality holds for all $f \in \mathcal{H}_{0}^{1}(\Omega)$, which is always true if $\Omega$ is bounded, but it also holds for some unbounded domains (see section I.7). The operator $G: \mathcal{H}^{-1}(\Omega) \rightarrow \mathcal{H}_{0}^{1}(\Omega)$ is defined by

$$
G f=\phi \text { if and only if } \phi \in \mathcal{H}_{0}^{1}(\Omega) \text { and }-\Delta \phi=f .
$$

Thus, in a certain sense, $G=-\Delta^{-1}$. We denote by $\gamma_{0}: \mathcal{H}^{1}(\Omega) \rightarrow \mathcal{H}^{\frac{1}{2}}(\Gamma)$ the Dirichlet trace operator, which is onto, and by $\gamma_{1}: \mathcal{H}^{2}(\Omega) \rightarrow \mathcal{H}^{\frac{1}{2}}(\Gamma)$ the outward normal derivative operator. Using Green's formula,

$$
\left\langle\gamma_{1} f, \gamma_{0} g\right\rangle_{L^{2}}=\langle\Delta f, g\rangle_{L^{2}}+\langle\nabla f, \nabla g\rangle_{L^{2}}
$$

for all $f \in \mathcal{H}^{2}(\Omega)$ and $g \in \mathcal{H}^{1}(\Omega), \gamma_{1}$ can be extended so that $\gamma_{1} f$ is defined as a distribution in $\mathcal{H}^{-\frac{1}{2}}(\Gamma)$ for every $f \in \mathcal{H}^{1}(\Omega)$ for which $\Delta f \in L^{2}(\Omega)$. Here, $\Delta f$ denotes the Laplacian of $f$ in the sense of distributions on $\Omega$, i.e., in the space $\mathcal{D}^{\prime}(\Omega)$.

We consider the system described by the equations

$$
\begin{gathered}
\ddot{z}=\Delta z \text { in } \Omega \times(0, \infty), \\
z=0 \quad \text { on } \Gamma_{0} \times(0, \infty), \\
z-\frac{1}{2} \gamma_{1}(G \dot{z})=u \text { on } \Gamma_{1} \times(0, \infty), \\
z(x, 0)=z_{0}(x), \quad \dot{z}(x, 0)=w_{0}(x) \text { for } x \in \Omega .
\end{gathered}
$$

The input of this system is the function $u$ in (5.31). The output associated with this system is

$$
y=z+\frac{1}{2} \gamma_{1}(G \dot{z}) \text { on } \Gamma_{1} \times(0, \infty) .
$$

Some comments about the domain $\Omega$ follow: It is not really necessary to assume that $\Gamma$ is of class $C^{2}$. What we really need is that $G$ maps $L^{2}(\Omega)$ onto $\mathcal{H}^{2}(\Omega) \cap \mathcal{H}_{0}^{1}(\Omega)$ and that $\gamma_{1}$ maps $\mathcal{H}^{2}(\Omega) \cap \mathcal{H}_{0}^{1}(\Omega)$ onto $\mathcal{H}^{\frac{1}{2}}(\Gamma)$. These properties hold also for some less regular domains, for example, for convex sets in $\mathbb{R}^{2}$ (see Grisvard $[9$, Theorem 3.2 .1 .2 , p. 147]) or for rectangular domains in $\mathbb{R}^{n}$. We will need the following simple result, a direct consequence of the Riesz representation theorem in $L^{2}(\Omega)$.

Proposition 5.5. For every $\mathrm{v} \in L^{2}(\Gamma)$ there exists a unique function $D \mathrm{v} \in$ $L^{2}(\Omega)$ such that

$$
\int_{\Omega}(D \mathrm{v})(x) \overline{\psi(x)} d x=-\int_{\Gamma} \mathrm{v} \overline{\gamma_{1}(G \psi)} d \Gamma \quad \forall \psi \in L^{2}(\Omega) .
$$

Moreover, the operator $D$ defined above (called the Dirichlet map) is linear and bounded from $L^{2}(\Gamma)$ into $L^{2}(\Omega)$.

Due to the Poincare inequality, the norm on $\mathcal{H}_{0}^{1}(\Omega)$ can be defined as $\|f\|_{\mathcal{H}_{0}^{1}}=$ $\|\nabla f\|_{L^{2}}$. Then it is easy to see (using (5.28)) that the corresponding dual norm on $\mathcal{H}^{-1}(\Omega)$ (with respect to the pivot space $L^{2}(\Omega)$ ) can be written as

$$
\|g\|_{\mathcal{H}^{-1}}=\sup _{\|f\|_{\mathcal{H}_{0}^{1}}=1}\langle g, f\rangle_{\mathcal{H}^{-1}, \mathcal{H}_{0}^{1}}=\|\nabla(G g)\|_{L^{2}}
$$


Define $W=\left\{f \in L^{2}(\Omega) \mid \Delta f \in \mathcal{H}^{-1}(\Omega)\right\}$. Since $\gamma_{1}$ maps $\mathcal{H}^{2}(\Omega) \cap \mathcal{H}_{0}^{1}(\Omega)$ onto $\mathcal{H}^{\frac{1}{2}}(\Gamma)$ (see Lions and Magenes [21, Chapter 2, Theorem 5.4]), the Dirichlet trace operator $\gamma_{0}$ can be extended to an operator $\gamma_{0}: W \rightarrow \mathcal{H}^{-\frac{1}{2}}(\Gamma)$ by putting

$$
\left\langle\gamma_{0} f, \gamma_{1}(G \psi)\right\rangle_{\mathcal{H}^{-\frac{1}{2}}, \mathcal{H}^{\frac{1}{2}}}=-\langle\Delta f, G \psi\rangle_{\mathcal{H}^{-1}, \mathcal{H}_{0}^{1}}-\langle f, \psi\rangle_{L^{2}} \quad \forall \psi \in L^{2}(\Omega) .
$$

The operators $D$ and $\gamma_{0}$ defined above are related as follows.

Proposition 5.6. If $\mathrm{v} \in L^{2}(\Gamma)$, then $\Delta(D \mathrm{v})=0$ in $\mathcal{D}^{\prime}(\Omega)$ (which implies that $D \mathrm{v} \in W)$. Moreover, we have that $\gamma_{0}(D \mathrm{v})=\mathrm{v}$.

Proof. The fact that $\Delta(D \mathrm{v})=0$ (in the sense of distributions) follows directly from (5.34) by taking $\psi=\Delta \varphi$, where $\varphi \in \mathcal{D}(\Omega)=C_{0}^{\infty}(\Omega)$. Now using the definition of the extended $\gamma_{0}$, we have that

$$
\left\langle\gamma_{0}(D \mathrm{v}), \gamma_{1}(G \psi)\right\rangle_{\mathcal{H}^{-\frac{1}{2}}, \mathcal{H}^{\frac{1}{2}}}=-\langle D \mathrm{v}, \psi\rangle_{L^{2}} \quad \forall \psi \in L^{2}(\Omega) .
$$

Since $\gamma_{1}(G \psi)$ in $(5.35)$ can be any function in $\mathcal{H}^{\frac{1}{2}}(\Gamma)$ and $\mathcal{H}^{\frac{1}{2}}(\Gamma)$ is dense in $L^{2}(\Omega)$, equations (5.34) and (5.35) imply that $\gamma_{0}(D \mathrm{v})=\mathrm{v}$.

To discuss the well-posedness and conservativity of the system (5.29)-(5.33) (using Theorem 1.1), we have to introduce the appropriate spaces and operators. Denote

$$
X=L^{2}(\Omega) \times \mathcal{H}^{-1}(\Omega), \quad U=L^{2}\left(\Gamma_{1}\right) .
$$

We also define the Hilbert space

$$
Z_{0}=\left\{f \in W \mid \gamma_{0} f \in L^{2}(\Gamma) \text { and }\left.\gamma_{0} f\right|_{\Gamma_{0}}=0\right\},
$$

with the norm $\|f\|_{Z_{0}}$ given by

$$
\|f\|_{Z_{0}}^{2}=\|f\|_{L^{2}}^{2}+\|\Delta f\|_{\mathcal{H}^{-1}}^{2}+\left\|\gamma_{0} f\right\|_{L^{2}}^{2} .
$$

The precise statement of the well-posedness and conservativity of the system described by (5.29)-(5.33) is given in the following proposition.

Proposition 5.7. The equations (5.29)-(5.33) determine a conservative linear system $\Sigma$ with input and output space $U$ and state space $X$. If $z_{0} \in Z_{0}, w_{0} \in$ $L^{2}(\Omega), u \in \mathcal{H}^{1}(0, \infty ; U)$, and the compatibility condition

$$
z_{0}(x)-\frac{1}{2} \gamma_{1}\left(G w_{0}\right)(x)=u(x, 0) \text { for } x \in \Gamma_{1}
$$

holds, then (5.29)-(5.33) have a unique solution $z$, y satisfying

$$
\begin{gathered}
z \in B C\left(0, \infty ; Z_{0}\right) \cap B C^{1}\left(0, \infty ; L^{2}(\Omega)\right) \cap B C^{2}\left(0, \infty ; \mathcal{H}^{-1}(\Omega)\right), \\
y \in \mathcal{H}^{1}(0, \infty ; U) .
\end{gathered}
$$

Proof. We define the following spaces and operators:

- The space $H$ is defined by $H=\mathcal{H}^{-1}(\Omega)$ endowed with the norm

$$
\|f\|_{H}=\|\nabla(G f)\|_{L^{2}(\Omega)} .
$$

- The operator $A_{0}: \mathcal{D}\left(A_{0}\right) \rightarrow H$ is defined by

$$
\mathcal{D}\left(A_{0}\right)=\mathcal{H}_{0}^{1}(\Omega), \quad A_{0} \phi=-\Delta \phi \quad \forall \phi \in \mathcal{D}\left(A_{0}\right) .
$$

It is well known that $A_{0}$ is self-adjoint, positive, and boundedly invertible. 
- As in section 1 , we put $H_{1}=\mathcal{D}\left(A_{0}\right)$ and we introduce the spaces $H_{\alpha}(\alpha \in \mathbb{R})$ by considering powers of $A_{0}$ and duality so that $A_{0}: H_{\alpha} \rightarrow H_{\alpha-1}$. In order to identify the space $H_{\frac{1}{2}}=\mathcal{D}\left(A_{0}^{\frac{1}{2}}\right)$, we recall that $H_{\frac{1}{2}}$ is the completion of $\mathcal{D}\left(A_{0}\right)$ with respect to the norm

$$
\|z\|_{\frac{1}{2}}=\left\|A_{0}^{\frac{1}{2}} z\right\|_{H}=\sqrt{\left\langle A_{0} z, z\right\rangle_{H}} .
$$

Since for any $z \in \mathcal{D}\left(A_{0}\right)=\mathcal{H}_{0}^{1}(\Omega)$, we have

$$
\left\langle A_{0} z, z\right\rangle_{H}=-\left\langle\nabla z, \nabla\left(\Delta^{-1} z\right)\right\rangle_{L^{2}(\Omega)}=\|z\|_{L^{2}(\Omega)}^{2} ;
$$

the space $H_{\frac{1}{2}}$ is given by $H_{\frac{1}{2}}=L^{2}(\Omega)$.

- Notice that $H_{\frac{3}{2}}=\mathcal{H}^{2}(\Omega) \cap \mathcal{H}_{0}^{1}(\Omega)$. We define the space

$$
\widetilde{\mathcal{H}}^{-2}(\Omega)=\left(\mathcal{H}^{2}(\Omega) \cap \mathcal{H}_{0}^{1}(\Omega)\right)^{\prime} .
$$

(This dual is computed with respect to the pivot space $L^{2}(\Omega)$.) It can be checked that $G$ can be extended so that $G: \widetilde{\mathcal{H}}^{-2}(\Omega) \rightarrow L^{2}(\Omega)$ and the norm on $\widetilde{\mathcal{H}}^{-2}(\Omega)$ is

$$
\|g\|_{\widetilde{\mathcal{H}}^{-2}}=\sup _{\|\Delta f\|_{L^{2}(\Omega)}=1}\langle g, f\rangle=\|G g\|_{L^{2}} .
$$

- By definition, the space $H_{-\frac{1}{2}}$ is the completion of $H$ with respect to the norm $\|z\|_{-\frac{1}{2}}=\left\|A_{0}^{-\frac{1}{2}} z\right\|_{H}$. Since $G=A_{0}^{-1}$ on $H$, the last equality and the definition of the norm on $H$ imply that

$$
\|z\|_{-\frac{1}{2}}=\left\|\nabla\left(A_{0}^{-\frac{3}{2}} z\right)\right\|_{L^{2}} .
$$

The above relation and the obvious fact that $\left\|A_{0}^{\frac{1}{2}} w\right\|_{L^{2}}=\|\nabla w\|_{L^{2}}$ for any $w \in \mathcal{H}_{0}^{1}(\Omega)$ imply that

$$
\|z\|_{-\frac{1}{2}}=\left\|A_{0}^{-1} z\right\|_{L^{2}}=\|G z\|_{L^{2}} \quad \forall z \in H .
$$

We have thus shown that $H_{-\frac{1}{2}}=\widetilde{\mathcal{H}}^{-2}(\Omega)$.

- We denote by $P \in \mathcal{L}\left(L^{2}(\Gamma), L^{2}\left(\Gamma_{1}\right)\right)$ the operator of truncation to $\Gamma_{1}$. Then $P^{*} \in \mathcal{L}\left(L^{2}\left(\Gamma_{1}\right), L^{2}(\Gamma)\right)$ is the operator defined by extending the functions in $L^{2}\left(\Gamma_{1}\right)$ by zero outside $\Gamma_{1}$. The operator $B_{0} \in \mathcal{L}\left(U, H_{-\frac{1}{2}}\right)$ is defined by

$$
B_{0} \mathrm{v}=A_{0} D P^{*} \mathrm{v},
$$

where $A_{0}$ is considered as an operator from $H_{\frac{1}{2}}$ to $H_{-\frac{1}{2}}$ and $D: U \rightarrow L^{2}(\Omega)$ is the Dirichlet map defined in Proposition 5.5.

- Let $\phi \in \mathcal{H}_{0}^{1}(\Omega)=H_{1}$ and $\mathrm{v} \in L^{2}\left(\Gamma_{1}\right)$. Then we have

$$
\left\langle B_{0} \mathrm{v}, \phi\right\rangle_{H_{-\frac{1}{2}}, H_{\frac{1}{2}}}=\left\langle D P^{*} \mathrm{v}, A_{0} \phi\right\rangle_{H}=\left\langle D P^{*} \mathrm{v}, \phi\right\rangle_{H_{\frac{1}{2}}} .
$$

By using the fact that $H_{\frac{1}{2}}=L^{2}(\Omega)$, the density of $\mathcal{H}_{0}^{1}(\Omega)$ in $L^{2}(\Omega)$, and (5.34), it follows that for every $\phi \in L^{2}(\Omega)$ and for every $\mathrm{v} \in L^{2}\left(\Gamma_{1}\right)$

$$
\left\langle B_{0} \mathrm{v}, \phi\right\rangle_{H_{-\frac{1}{2}}, H_{\frac{1}{2}}}=\left\langle D P^{*} \mathrm{v}, \phi\right\rangle_{L^{2}}=-\int_{\Gamma_{1}} \mathrm{v} \overline{\gamma_{1}(G \phi)} \mathrm{d} \Gamma .
$$


We conclude that the adjoint of $B_{0}$ (using the pivot space $H=\mathcal{H}^{-1}(\Omega)$ ) is $C_{0} \in \mathcal{L}\left(H_{\frac{1}{2}}, U\right)$ given by

$$
C_{0} \phi=B_{0}^{*} \phi=-P \gamma_{1}(G \phi) \quad \forall \phi \in H_{\frac{1}{2}}=L^{2}(\Omega) .
$$

It is clear that the spaces $U, H, H_{\alpha}, X$ and the operators $A_{0}, C_{0}, B_{0}$ fit into the simple general framework of section 1 . Thus, by Theorem 1.1, they determine via the equations (1.1)-(1.3) a conservative linear system $\Sigma$. The state of this system is

$$
\xi(t)=\left[\begin{array}{c}
z(t) \\
\dot{z}(t)
\end{array}\right]
$$

To show that $\Sigma$ is described by (5.29)-(5.33), first we notice that the space $Z_{0}=$ $H_{1}+A_{0}^{-1} B_{0} U$ defined after (1.5) (see also Theorem I.1.2) is given in our case by $Z_{0}=H_{1}+D P^{*} U$. By using Lemma 5.6, it can be checked that this space coincides with that defined by (5.36). We define the operator $G_{0} \in \mathcal{L}\left(Z_{0}, U\right)$ by

$$
G_{0} f=P \gamma_{0} f \quad \forall f \in Z_{0} .
$$

Clearly, we have $G_{0} H_{1}=\{0\}$, and by Lemma 5.6 we have in $\mathcal{L}(U)$

$$
G_{0} A_{0}^{-1} B_{0}=P \gamma_{0} A_{0}^{-1}\left(A_{0} D P^{*}\right)=P \gamma_{0} D P^{*}=I .
$$

Hence, all the assumptions in Theorem I.1.4 are satisfied. We define on $Z_{0}$ the operator $L_{0}=A_{0}-B_{0} G_{0}$ as in Theorem I.1.4, and it is easy see that $L_{0} z=-\Delta z$.

If we write the system of equations (I.1.16) in our specific framework, we obtain the system of equations (5.29)-(5.33). Hence, by Theorem I.1.4 the compatibility condition in our proposition is equivalent to (I.1.9), and the equations (5.29)-(5.33) are equivalent to (I.1.11) and (I.1.12). Now by Theorem I.1.2, the equations have a solution $z, y$ with the claimed smoothness properties.

This system has also been considered in Lasiecka and Triggiani [18, pp. 669-671] but without considering outputs. They have proved the well-posedness of the mapping from the input function to the state, and they have discussed the exponential stability of the system for suitable $\Gamma_{1}$.

THEOREM 5.8. If $\Omega$ is bounded, then the system defined by (5.29)-(5.33) is strongly stable, exactly controllable in infinite time, and exactly observable in infinite time.

Proof. The fact that the equations (5.29)-(5.33) define a conservative linear system with input space $U$, state space $X$, and output space $Y$ has been said in Proposition 5.7. The boundedness of $\Omega$ implies that the spectrum of $A_{0}$ is countable. Thus, according to assertion (8) of Theorem 1.5, in order to check the properties claimed in the theorem it suffices to prove that for any $\phi \in H_{1}$ if $\phi$ is an eigenvector of $A_{0}$, then $C_{0} \phi \neq 0$. Due to the particular form of $A_{0}$ and $C_{0}$, this means that we have to show that if $\phi \in \mathcal{H}_{0}^{1}(\Omega)$ is such that for some $\lambda>0$

$$
-\Delta \phi=\lambda \phi \text { in } \mathcal{H}^{-1}(\Omega), \quad \frac{\partial}{\partial \nu}(G \phi)=0 \text { on } \Gamma_{1},
$$

then $\phi=0$. By denoting $\psi=G \phi$, we see that (5.37) is equivalent to

$$
-\Delta \psi=\lambda \psi \text { in } \mathcal{H}_{0}^{1}(\Omega), \quad \frac{\partial \psi}{\partial \nu}=0 \text { on } \Gamma_{1} .
$$


By classical elliptic regularity results, (5.38) implies that $\psi \in \mathcal{H}^{2}(\Omega)$ (see, for instance, Grisvard [9, Theorem 2.2.2.5]). This fact combined with (5.38) implies, by a classical unique continuation argument (see, for instance, Komornik [16, Corollary $6.2]$ ), that $\psi=0$. Thus, (5.37) implies that $\phi=\Delta \psi=0$ in $\mathcal{H}_{0}^{1}(\Omega)$.

The exponential stability of the system (5.29)-(5.32) was studied in Bardos et al. [4]. By combining Proposition 5.7 and [4, Theorem 1], we obtain the following.

TheOREM 5.9. Suppose that $\Omega$ is bounded and there exists a time $T_{0}>0$ such that every geometric ray in $\Omega \times\left(0, T_{0}\right)$ intersects $\Gamma_{1} \times\left(0, T_{0}\right)$ in a nondiffractive point. Then the equations (5.29)-(5.33) define an exponentially stable, conservative system with input space $U$, state space $X$, and output space $Y$. This system is also exactly controllable and exactly observable in time $T_{0}$.

The last sentence of the above theorem follows from the exponential stability (stated in the first part of the theorem) using Theorem 1.3.

Acknowledgments. We have had useful discussions on this research with Olof Staffans, Arjan van der Schaft, and Peng-Fei Yao.

\section{REFERENCES}

[1] K. Ammari, M. Tucsnak, And Z. Liu, Decay rates for a beam with pointwise force and moment feedback, Math. Control Signals Systems, 15 (2002), pp. 229-255.

[2] W. Arendt And C.J.K. Batty, Tauberian theorems and stability of one-parameter semigroups, Trans. Amer. Math. Soc., 306 (1988), pp. 837-841.

[3] S.A. Avdonin And S.A. Ivanov, Families of Exponentials, Cambridge University Press, Cambdridge, UK, 1995.

[4] C. Bardos, L. Halpern, G. Lebeau, J. Rauch, and E. Zuazua, Stabilisation de l'équation des ondes au moyen d'un feedback portant sur la condition aux limites de Dirichlet, Asymptot. Anal., 4 (1991), pp. 285-291.

[5] C. Bardos, G. Lebeau, and J. Rauch, Sharp sufficient conditions for the observation, control and stabilization of waves from the boundary, SIAM J. Control. Optim., 30 (1992), pp. 1024-1065.

[6] A. Bensoussan, G. Da Prato, M.C. Delfour, and S.K. Mitter, Representation and Control of Infinite Dimensional Systems, Vol. 1, Birkhäuser, Boston, 1992.

[7] P.L. Butzer And H. Berens, Semi-Groups of Operators and Approximation, Grundlehren Math. Wiss. 145, Springer-Verlag, New York, 1967.

[8] P. Grabowski, On the spectral Lyapunov approach to parametric optimization of distributed parameter systems, IMA J. Math. Control Inform., 7 (1990), pp. 317-338.

[9] P. Grisvard, Elliptic Problems in Nonsmooth Domains, Monogr. Stud. Math. 24, Pitman, Boston, 1985.

[10] B.-Z. Guo AND Y.-H. LuO, Controllability and stability of a second-order hyperbolic system with collocated sensor/actuator, Systems Control Lett., 46 (2002), pp. 45-65.

[11] B.-Z. GuO AND Y.-H. LuO, Riesz basis property of a second-order hyperbolic system with collocated scalar input-output, IEEE Trans. Automat. Control, 47 (2002), pp. 693-698.

[12] S. Hansen and G. Weiss, New results on the operator Carleson measure criterion, IMA J. Math. Control Inform., 14 (1997), pp. 3-32.

[13] H. Falun, Characteristic conditions for exponential stability of linear dynamical systems in Hilbert spaces, Ann. Differential Equations, 1 (1985), pp. 43-56.

[14] B. Jacob and J. Partington, The Weiss conjecture on admissibility of observation operators for contraction semigroups, Integral Equations Operator Theory, 40 (2001), pp. 231-243.

[15] B. JACOB AND H. ZWART, Exact observability of diagonal systems with a finite dimensional output operator, Systems Control Lett., 43 (2001), pp. 101-109.

[16] V. Komornik, Exact Controllability and Stabilization - The Multiplier Method, John Wiley, Chichester, UK, Masson, Paris, 1994.

[17] J.E. Lagnese and J.-L. Lions, Modelling, Analysis and Control of Thin Plates, Masson, Paris, Springer-Verlag, Berlin, 1988.

[18] I. Lasiecka and R. Triggiani, Control Theory for Partial Differential Equations: Continuous and Approximation Theories, Cambridge University Press, Cambridge, UK, 2000. 
[19] G. LeBeAu, Equation des ondes amorties, in Algebraic and Geometric Methods in Mathematical Physics, A. Boutet de Monvel and V. Marchenko, eds., Kluwer Academic Publishers, Dordrecht, The Netherlands, 1996, pp. 73-109.

[20] J.-L. Lions, Controlabilité Exacte Perturbations et Stabilisation de Systèmes Distribués, Vol. 1, Masson, Paris, 1988.

[21] J.-L. Lions And E. Magenes, Non-Homogeneous Boundary Value Problems and Applications, Vol. I, Grundlehren Math. Wiss. 181, Springer-Verlag, Berlin, 1972.

[22] K. LIU, Locally distributed control and damping for the conservative systems, SIAM J. Control Optim., 35 (1997), pp. 1574-1590.

[23] K. LiU, Z. LIU, AND B. RAO, Exponential stability of an abstract nondissipative linear system, SIAM J. Control Optim., 40 (2001), pp. 149-165.

[24] A. PAZY, Semigroups of Linear Operators and Applications to Partial Differential Equations, Springer-Verlag, New York, 1983.

[25] J. Prüss, On the spectrum of $C_{0}$-semigroups, Trans. Amer. Math. Soc., 284 (1984), pp. $847-$ 857.

[26] R. Rebarber, Exponential stability of coupled beams with dissipative joints: A frequency domain approach, SIAM J. Control Optim., 33 (1995), pp. 1-28.

[27] R. Rebarber and G. Weiss, Necessary conditions for exact controllability with a finitedimensional input space, Systems Control Lett., 40 (2000), pp. 217-227.

[28] D.L. Russell and G. Weiss, A general necessary condition for exact observability, SIAM J. Control Optim., 32 (1994), pp. 1-23.

[29] M. TUCSNAK AND G. WeIss, Simultaneous exact controllability and some applications, SIAM J. Control Optim., 38 (2000), pp. 1408-1427.

[30] G. WEISS, Weak $L^{p}$-stability of a linear semigroup on a Hilbert space implies exponential stability, J. Differential Equations, 76 (1988), pp. 269-285.

[31] G. WeIss, Transfer functions of regular linear systems. Part I: Characterizations of regularity, Trans. Amer. Math. Soc., 342 (1994), pp. 827-854.

[32] G. WeIss, Regular linear systems with feedback, Math. Control Signals Systems, 7 (1994), pp. $23-57$.

[33] G. Weiss and R. Rebarber, Optimizability and estimatability for infinite-dimensional linear systems, SIAM J. Control Optim., 39 (2000), pp. 1204-1232.

[34] G. Weiss, O.J. Staffans, and M. Tucsnak, Well-posed linear systems-a survey with emphasis on conservative systems, Int. J. Appl. Math. Comput. Sci., 11 (2001), pp. 7-33.

[35] G. Weiss AND M. Tucsnak, How to get a conservative well-posed linear system out of thin air. Part I. Well-posedness and energy balance, ESAIM Control Optim. Calc. Var., 9 (2003), pp. 247-274. 